ARTICLE

DOI: $10.1038 / s 41467-018-04176-x$

\title{
Defining a conformational ensemble that directs activation of PPAR $\gamma$
}

Ian M. Chrisman 1,2, Michelle D. Nemetchek ${ }^{1,2,3}$, Ian Mitchelle S. de Vera ${ }^{4,8}$, Jinsai Shang ${ }^{4}$, Zahra Heidari ${ }^{2,3}$, Yanan Long (10 4,5, Hermes Reyes-Caballero ${ }^{4}$, Rodrigo Galindo-Murillo (1) ${ }^{6}$, Thomas E. Cheatham, III6, Anne-Laure Blayo7, Youseung Shin ${ }^{7}$, Jakob Fuhrmann ${ }^{4}$, Patrick R. Griffin ${ }^{4,7}$, Theodore M. Kamenecka7, Douglas J. Kojetin (1) ${ }^{4,7} \&$ Travis S. Hughes (1) ${ }^{2,3}$

The nuclear receptor ligand-binding domain (LBD) is a highly dynamic entity. Crystal structures have defined multiple low-energy LBD structural conformations of the activation function-2 (AF-2) co-regulator-binding surface, yet it remains unclear how ligand binding influences the number and population of conformations within the AF- 2 structural ensemble. Here, we present a nuclear receptor co-regulator-binding surface structural ensemble in solution, viewed through the lens of fluorine-19 $\left({ }^{19} \mathrm{~F}\right)$ nuclear magnetic resonance (NMR) and molecular simulations, and the response of this ensemble to ligands, co-regulator peptides and heterodimerization. We correlate the composition of this ensemble with function in peroxisome proliferator-activated receptor- $\gamma$ (PPAR $\gamma$ ) utilizing ligands of diverse efficacy in co-regulator recruitment. While the co-regulator surface of apo PPAR $\gamma$ and partial-agonistbound PPAR $\gamma$ is characterized by multiple thermodynamically accessible conformations, the full and inverse-agonist-bound PPAR $\gamma$ co-regulator surface is restricted to a few conformations which favor coactivator or corepressor binding, respectively.

\footnotetext{
${ }^{1}$ Biochemistry and Biophysics Graduate Program, The University of Montana, Missoula, MT 59812, USA. ${ }^{2}$ Center for Biomolecular Structure and Dynamics, The University of Montana, Missoula, MT 59812, USA. ${ }^{3}$ Department of Biomedical and Pharmaceutical Sciences, The University of Montana, Missoula, MT 59812, USA. ${ }^{4}$ Department of Integrative Structural and Computational Biology, Scripps Florida, The Scripps Research Institute, Jupiter, FL 33458 , USA.

${ }^{5}$ Summer Undergraduate Research Fellows (SURF) Program, Scripps Florida, The Scripps Research Institute, Jupiter, FL 33458, USA. ${ }^{6}$ Department of Medicinal Chemistry, University of Utah, Salt Lake City, UT 84112, USA. ${ }^{7}$ Department of Molecular Medicine, Scripps Florida, The Scripps Research Institute, Jupiter, FL 33458, USA. ${ }^{8}$ Present address: Department of Pharmacology \& Physiology, Saint Louis University School of Medicine, Saint Louis, MO 63104, USA. These authors contributed equally: Michelle D. Nemetchek, lan Mitchelle S. de Vera, Jinsai Shang. Correspondence and requests for materials should be addressed to T.S.H. (email: travis.hughes@umontana.edu)
} 
$\mathrm{N}$ uclear receptors are ligand-regulated transcription factors that mediate the transcriptional actions of lipophilic endogenous ligands, including steroid hormones and lipids ${ }^{1}$, and are the target of $\sim 13 \%$ of US Food and Drug Administration (FDA)-approved drugs ${ }^{2}$. The binding of these natural ligands, as well as synthetic ligands and FDA-approved drugs, to the nuclear receptor ligand-binding domain (LBD) affects the recruitment of transcriptional co-regulator proteins to target gene promoters, which influences chromatin remodeling and gene transcription ${ }^{3}$. Crystal structures of nuclear receptor LBDs have revealed in exquisite detail the molecular contacts created between the receptor and ligand, as well as low-energy active and inactive conformations of helix $12^{4-7}$. Helix 12 is a critical regulatory structural element in the activation function-2 (AF-2) co-regulator interaction surface of many nuclear receptors $^{8}$. Over 100 crystal structures have been solved of the peroxisome proliferator-activated receptor- $\gamma$ (PPAR $\gamma$ ) LBD bound to ligands of various scaffolds and pharmacological activities ${ }^{9}$. Surprisingly, the backbone conformations of these structures, in particular the conformation of helix 12, are all very similar despite the fact that PPAR $\gamma$ is bound to ligands that produce a diverse range of functional outputs. Thus, it is difficult to understand the structural mechanism of action by which the binding of ligands with diverse activities affect helix 12 conformation from crystallography data alone. One hypothesis is that helix 12 consists of a dynamic ensemble of conformations, and not one single or static conformation in the presence or absence of a bound ligand ${ }^{10,11}$. However, experimental evidence describing this ensemble is lacking and it remains poorly understood how binding of pharmacologically distinct ligands affects the ensemble of co-regulator-binding surface and helix 12 conformations.

Solution structural methods indicate prevalent, liganddependent helix 12 movement. Hydrogen deuterium exchange mass spectrometry (HDX-MS) demonstrates a relationship between helix 12 stability and agonist binding for nuclear receptors $^{12-15}$. Nuclear magnetic resonance (NMR) studies implicate movement on the microsecond-millisecond $(\mu \mathrm{s}-\mathrm{ms})$ time scale between two or more conformations over a large portion of the apo PPAR $\gamma$ LBD and partial-agonist-bound PPAR $\gamma$ LBD. These movements result in very broad or unobserved NMR resonances that prohibit structural analyses. Full agonists robustly diminish these dynamics ${ }^{16-19}$. Furthermore, crystal structures, HDX-MS, and protein NMR have provided complementary information revealing a relationship between structure and function for PPAR $\gamma$ (e.g., the presence or absence of critical hydrogen bonds between ligand and helix $12^{20}$ ); however, a direct observation of the ligand-dependent ensemble implied by these data is lacking. This raises the question: are there multiple long-lived conformations that correlate with functional efficacy (e.g., co-regulator affinity) in nuclear receptors?

It remains challenging to quantify the number, relative population, and kinetics of exchange between the conformations that compose this putative ensemble and how the ensemble is influenced by binding small molecules and co-regulators. ${ }^{19} \mathrm{~F}$ (fluorine-19) NMR spectroscopy is exceptionally sensitive to structural and environmental changes, can reveal structural information from regions of a protein that are unobserved via $2 \mathrm{D} / 3 \mathrm{D} N \mathrm{NR}^{21}$, and can be used to probe how ligands affect the conformational ensemble of proteins ${ }^{22-26}$. Here, using ${ }^{19} \mathrm{~F}$ NMR combined with biochemical co-regulator interaction analysis and molecular simulations, we define the ligand-dependent conformational ensemble of the co-regulator interaction surface, including helix 12 , which controls the transcriptional activity of PPAR $\gamma$. The data presented here indicate that helix 12 and the co-regulator-binding surface of apo PPAR $\gamma$ and partial-agonist-bound PPAR $\gamma$ is found in a broad energy well with multiple local minima of similar potential energy separated by relatively small kinetic barriers, allowing exchange on the $\mu$ s to $\mathrm{ms}$ time scale. In contrast, when PPAR $\gamma$ is bound to a full agonist or inverse agonist, helix 12 and the co-regulator-binding surface occupies narrow energy wells with fewer thermodynamically accessible conformations. In addition, simulations define some of the probable structures that compose these ensembles. These data better elucidate how ligands induce functional effects via nuclear receptors.

\section{Results}

Diverse activities of synthetic PPAR $\gamma$ ligands. We assembled a set of 16 pharmacologically distinct PPAR $\gamma$ ligands that we and others developed or previously characterized in cellular and structure-function studies (Supplementary Fig. 16) including full and partial agonists that robustly or mildly enhance transcriptional activation; antagonists/non-agonists that block activation or maintain constitutive basal cellular transcriptional activity; and inverse agonists that repress transcription compared to the basal receptor activity. In a time-resolved fluorescence resonance energy transfer (TR-FRET) co-regulator interaction assay, full agonists such as GW1929 and rosiglitazone that induce robust PPAR $\gamma$ transcription ${ }^{27,} 28$ increase binding of a peptide derived from mediator of RNA polymerase II transcription subunit 1 (MED1) coactivator (Fig. 1a), and decrease binding of a peptide derived from the nuclear receptor corepressor 1 (NCoR1) referred to herein as NCoR (Fig. 1b). In contrast, ligands that function as inverse agonists such as T0070907 and SR10221 lower PPAR $\gamma$ transcriptional responses relative to basal cellular activity ${ }^{13,29,30}$, increase binding of NCoR (Fig. 1b), and decrease binding of MED1 (Fig. 1a). Crystal structures of ligand-bound PPAR $\gamma$ LBD typically show a non-crystallographic dimer configuration containing two chains, A and B (Supplementary Fig. 2a). The main difference between the two chain $A$ and chain $B$ protein molecules involves helix 12, which adopts distinct conformations commonly referred to as active and inactive. The active conformation is assumed to be the conformation in solution when bound to a full agonist that induces increased transcription. Notably, these active and inactive helix 12 conformations are both influenced by crystal contacts (Supplementary Fig. 2b).

NMR-detected co-regulator-binding surface structural ensemble. To facilitate ${ }^{19} \mathrm{~F}$ NMR studies of the co-regulator-binding surface, which is composed of portions of helix 3,4 , and 12 , we introduced a cysteine residue on the $\mathrm{C}$ terminus of helix 12 at several locations (K502C, Y505C, and Q498C) and on helix 3 (Q322C) of the PPAR $\gamma$ LBD to allow covalent linkage of 3bromo-1,1,1-trifluoroacetone (BTFA), a small molecule containing a trifluoromethyl $\left(-\mathrm{CF}_{3}\right)$ group. Q498C caused protein instability, whereas BTFA attached to Y505C did not show pronounced ligand-induced changes to the ${ }^{19} \mathrm{~F}$ NMR spectra of the PPAR $\gamma$ LBD, presumably due to its position at the unstructured C terminus of helix 12 (Supplementary Fig. 1). However, Q322C and $\mathrm{K} 502 \mathrm{C}$ yielded well-functioning protein with pronounced ligand-inducible changes; we used these mutants to probe the conformational ensemble of the PPAR $\gamma$ co-regulator-binding surface (i.e., the AF-2 surface).

Molecular simulations indicate that K502 (wild-type (wt) residue) and K502C-BTFA (modified residue) are both solvent exposed in the active helix 12 conformation and are not likely to sterically hinder co-regulator binding in this active conformation (Fig. 2a, Supplementary Fig. 3). Control experiments indicate that the introduced cysteine on helix 12 (C502) is preferentially labeled over the only native cysteine (C313), which could be because $\mathrm{C} 313$ points into the ligand-binding pocket 

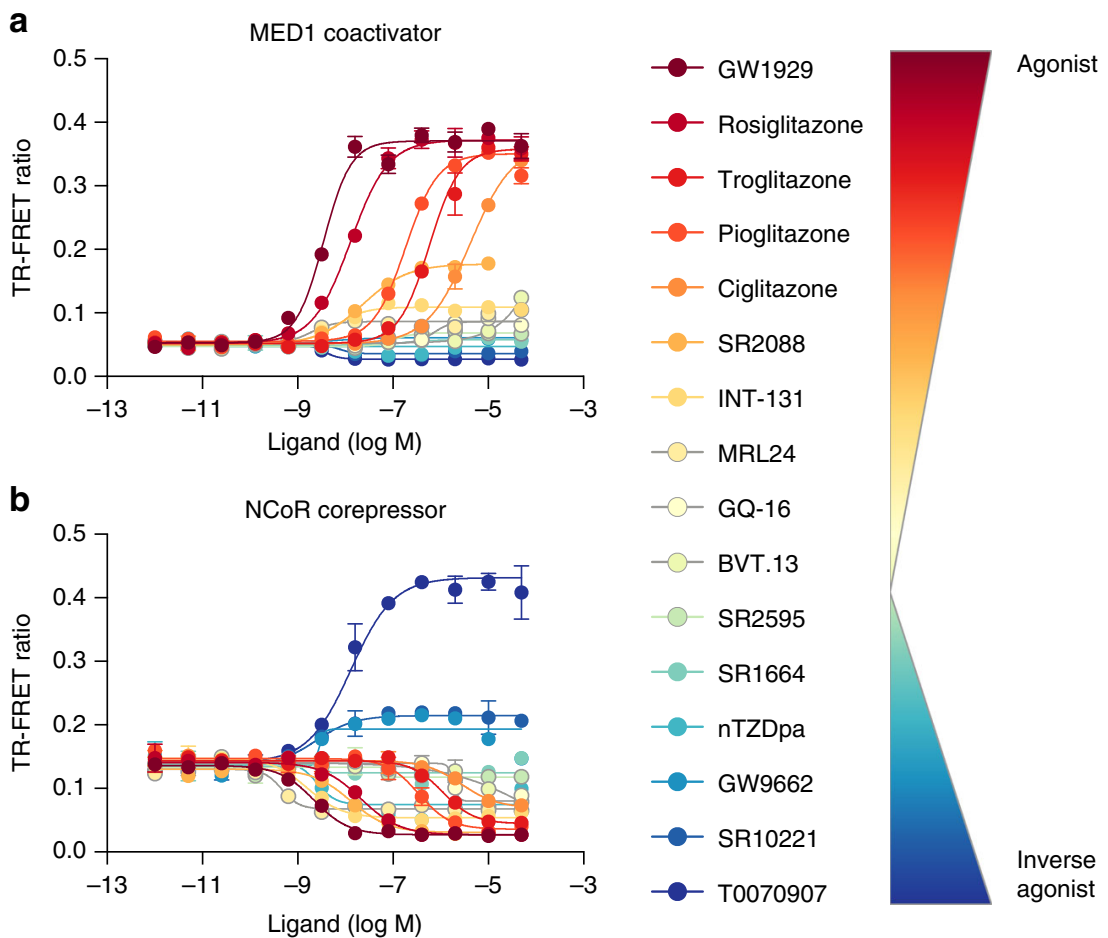

Fig. 1 Nuclear receptor co-regulator interaction differentiates pharmacologically distinct PPAR ligands. TR-FRET biochemical assay shows the effect of the compounds on the interaction between PPAR $\mathrm{LBD}$ and peptides derived from the $\mathbf{a}$ MED1 coactivator and $\mathbf{b}$ NCoR corepressor, plotted as TR-FRET ratio $(665 \mathrm{~nm} / 620 \mathrm{~nm})$ vs. ligand concentration $(n=2$, standard deviation). The data shown represents technical replicates from a single experiment and the experiment was repeated four times with similar results. The window of efficacy in these data is representative of ligand-induced changes in co-regulator affinity for PPAR $\gamma$. An increase in TR-FRET ratio indicates a strengthening of affinity for MED1/NCoR compared to apo while a decrease indicates a ligandinduced weakening of affinity for the co-regulator. The effect of vehicle (DMSO) is negligible (Supplementary Fig. 5); furthermore, the DMSO concentration is constant across the titration both in this figure and all other TR-FRET data presented

(Supplementary Fig. 4). We refer hereafter to BTFA-labeled PPAR $\gamma$ K502C protein as PPAR $\gamma^{\text {K502C }}$-BTFA, we also use PPAR $\gamma^{\text {K502C }}$-BTFA with a C313A mutation (referred to as

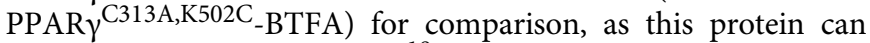
only be labeled on helix $12 .{ }^{19} \mathrm{~F}$ NMR signals in these labeled proteins could conceivably arise from BTFA-labeled co-purified protein impurities; however, the spectrum of PPAR $\gamma \mathrm{C} 313 \mathrm{~A}$, which lacks all cysteines, reveals no detectable signal from impurities (Supplementary Fig. 5).

Next, we determined the effects of mutations and labeling on the function of the PPAR $\gamma$ LBD. First, we measured ligand $K_{\mathrm{i}}$ values using a competitive ligand displacement assay to determine if the mutations and BTFA label affects ligand affinity. Compared to wt, the PPAR $\gamma^{\text {K502C }}$-BTFA and PPAR $\gamma^{\mathrm{C} 313 \mathrm{~A}, \mathrm{~K} 502 \mathrm{C}_{-\mathrm{BTFA}}}$ proteins exhibit a 5-fold and 11-fold median reduction in ligand affinity, respectively (Supplementary Table 1); however, only four ligands (GQ-16, BVT.13, ciglitazone, and troglitazone) are predicted to be at $<93 \%$ occupancy in our samples under the conditions used for the NMR experiments reported here (Supplementary Table 2). Second, we measured co-regulator recruitment efficacy and half-maximal effective concentration $\left(\mathrm{EC}_{50}\right)$ using TR-FRET. As expected from the calculated $K_{\mathrm{i}}$ values, PPAR ${ }^{\mathrm{K} 502 \mathrm{C}}$-BTFA has a 2-fold and 5-fold median reduction in $\mathrm{EC}_{50}$ and PPAR $\gamma^{\mathrm{C} 313 \mathrm{~A}, \mathrm{~K} 502 \mathrm{C}}$-BTFA has a 1-fold and 9-fold median reduction in $\mathrm{EC}_{50}$ in recruiting MED1 and $\mathrm{NCoR}$, respectively, compared to wt PPAR $\gamma$ (Supplementary Table 3). Importantly, ligand-induced co-regulator recruitment efficacy is highly correlated for PPAR $\gamma^{\mathrm{K} 502 \mathrm{C}}-\mathrm{BTFA}$ and $w t$, indicating that PPAR $\gamma$ K502C-BTFA is functionally similar to wt PPAR $\gamma$ LBD $\left(R^{2}\right.$ $=0.8$ for NCoR and $R^{2}=0.98$ for MED1; Supplementary Fig. $6 \mathrm{~d}$, f). In contrast, the relative NCoR recruitment efficacy differs between PPAR $\gamma^{\mathrm{C} 313 \mathrm{~A}, \mathrm{~K} 502 \mathrm{C}}-\mathrm{BTFA}$ and $\mathrm{wt}$, while the relative MED1 recruitment efficacy is comparable to wt in this labeled mutant $\left(R^{2}=0.11\right.$ for NCoR and $R^{2}=0.8$ for MED1; Supplementary Fig. 6d, f). We also found that the mutations and labeling had little effect on recruitment efficacy and $\mathrm{EC}_{50}$ of a coactivator peptide derived from CREB-binding protein (CBP) for both labeled forms of PPAR $\gamma$ (Supplementary Fig. 6b, e and Supplementary Table 4).

In addition, we compared mutant and wt PPAR $\gamma$ affinity for fluorescein isothiocyanate (FITC)-labeled NCoR, MED1, and a peptide from the silencing mediator of retinoic acid and thyroid hormone receptor (SMRT) corepressor utilizing fluorescence polarization (FP). Labeling did not significantly affect affinity of NCoR or MED1 for the four tested PPAR $\gamma$-ligand complexes (Supplementary Fig. 7), and consistent with the TR-FRET data, PPAR $\gamma^{\text {K502C }}$-BTFA affinity for NCoR and MED1 correlated most closely with wt PPAR $\gamma$. Both labeled PPAR $\gamma$ s affect SMRT peptide affinity, although PPAR $\gamma^{\mathrm{K} 502 \mathrm{C}}$-BTFA does so to a lesser extent, indicating that the label may directly interfere with SMRT binding (Supplementary Fig. 7). In general, these data indicate that the relative co-regulator affinities are consistent between wt and especially PPAR $\gamma^{\text {K502C }}$-BTFA. Given these data, we focused on PPAR $\gamma^{\mathrm{K} 502 \mathrm{C}}$-BTFA to correlate structure with function and utilize PPAR $\gamma^{\mathrm{C} 313 \mathrm{~A}, \mathrm{~K} 502 \mathrm{C}}-\mathrm{BTFA}$ to confirm specific labeling of PPAR $\gamma^{\mathrm{K} 502 \mathrm{C}}-\mathrm{BTFA}$ and in cases where increased signal to noise is required. PPAR $\gamma^{\mathrm{K} 502 \mathrm{C}}$ - BTFA is incompletely labeled (Supplementary Fig. 4d) to avoid labeling C313 (Supplementary Fig. $4 \mathrm{a}-\mathrm{c}$ ), which decreases the NMR signal. In addition, PPAR $\gamma$ K502C that is not labeled with BTFA is not TRFRET active (Supplementary Fig. 4e), but has the same affinity for co-regulators as PPAR $\gamma^{\mathrm{K} 502 \mathrm{C}_{-B T F A}}$ (Supplementary Fig. 7b); 
a

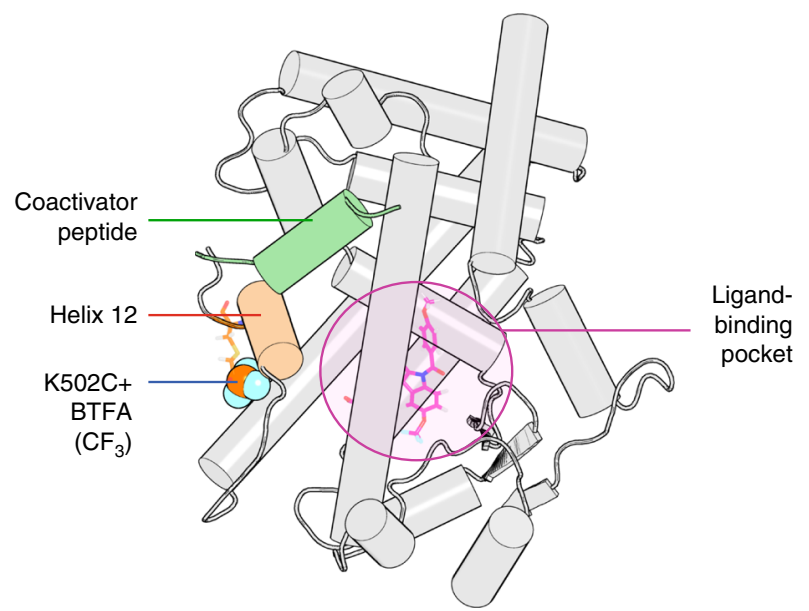

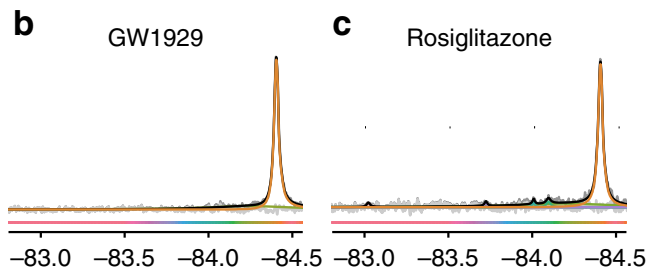

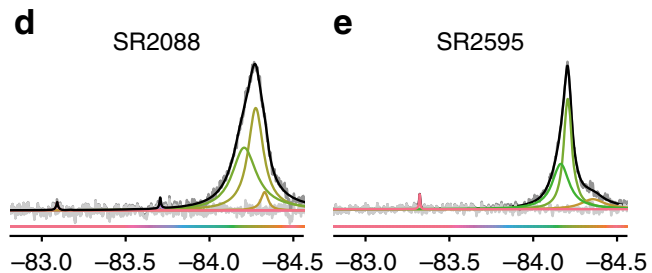

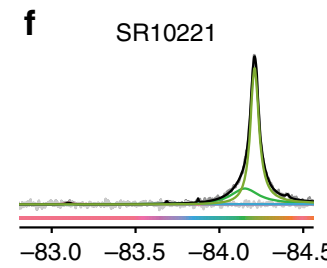

g Pioglitazone
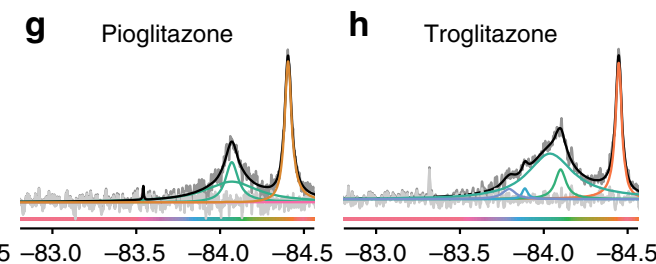

i nTZDpa

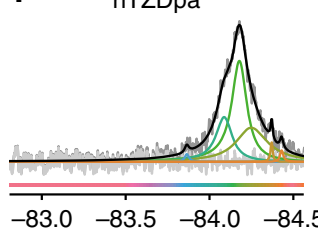

j Ciglitazone

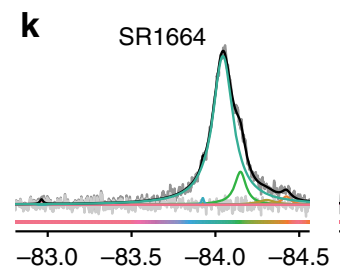

I BVT.13

m INT-131
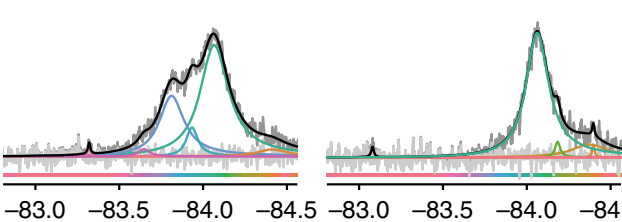

n GQ-16
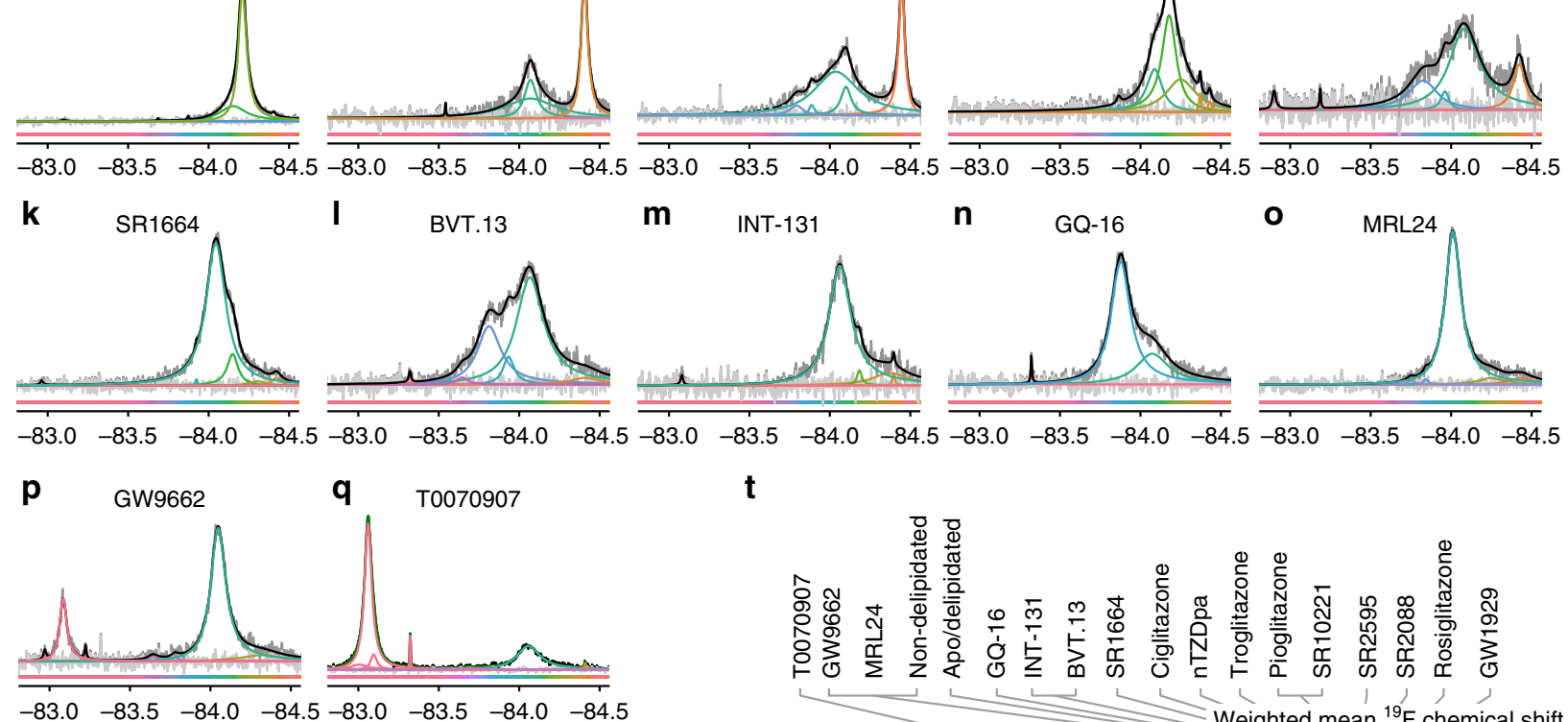

t
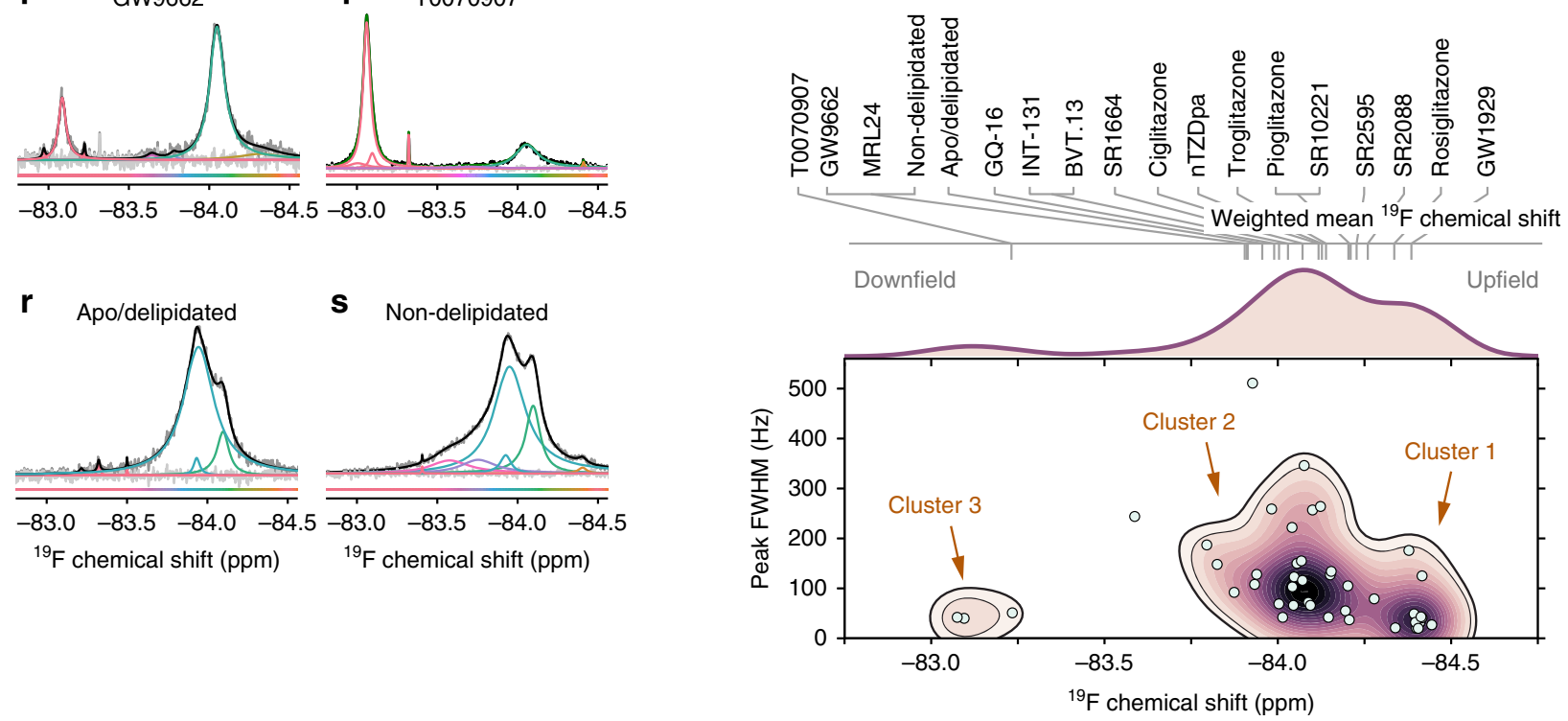

Fig. $2{ }^{19} \mathrm{~F}$ NMR analysis of PPAR $\gamma^{\mathrm{K} 502 \mathrm{C}}$-BTFA bound to 16 pharmacologically distinct ligands. a Location of covalently attached BTFA tag. b-s ${ }^{19} \mathrm{~F}$ NMR spectra (medium gray lines) of PPAR ${ }^{K 502 C}$-BTFA $\mathbf{b}-\mathbf{q}$ bound to ligand and ordered according to mean-weighted ${ }^{19} \mathrm{~F}$ chemical shift or $\mathbf{r}$ delipidated and $\mathbf{s}$ non-delipidated apoprotein. Fitted peaks are colored according to fitted ${ }^{19} \mathrm{~F}$ chemical shifts; deconvoluted spectra and residuals are shown in black and light gray lines, respectively. Some of these spectra have been replicated (Supplementary Fig. 10). $\mathbf{t}$ Plot of the full width half max (FWHM) of the major fitted peaks (only peaks that comprise $>5 \%$ of the spectrum area are included) vs. fitted chemical shifts. Clusters were detected by bivariate kernel density estimation (purple contours) with mean-weighted ${ }^{19} \mathrm{~F}$ chemical shift values annotated on top of the plot. Computational simulations were run of labeled PPARy (PPAR $\gamma^{K 502 C}$-BTFA) bound to one of these 16 ligands (GW1929) that demonstrate that the tag stays on or near the surface of the protein (Supplementary Fig. 3) 
therefore, any unlabeled portion of PPAR $\gamma^{\mathrm{K} 502 \mathrm{C}}$-BTFA should not affect FP or TR-FRET results.

We collected ${ }^{19} \mathrm{~F}$ NMR spectra of PPAR $\gamma^{\mathrm{K} 502 \mathrm{C}}$-BTFA bound individually to each of the 16 synthetic ligands (Fig. $2 \mathrm{~b}-\mathrm{q}$ ), as well as spectra of apoprotein with and without delipidation (Fig. 2r, s). Bacterially expressed PPAR $\gamma$ often contains fatty acids, which can have functional effects ${ }^{31}$, and therefore we remove these lipids; additional data regarding the effects of delipidation on structure and function are presented below. ${ }^{19} \mathrm{~F}$ NMR provides a time-averaged view of the conformational ensemble of helix 12, thus long-lived major conformations ( $>$ ms lifetime) show up as distinct peaks, while conformations with lifetimes on the $\mu \mathrm{s}-\mathrm{ms}$ time scale show up as a broad single peak. A single conformation (i.e., fast exchange among minor conformational variants) produces a single narrow peak. We used an objective deconvolution $^{32}$ method to determine the number of peaks and corresponding peak line widths that compose the recorded spectra. The line widths of the peaks obtained from the objective deconvolution are consistent with measured NMR lifetimes of the ${ }^{19} \mathrm{~F}\left(T_{2}\right)$ that we obtained for several liganded states (Supplementary Table 5). Bivariate kernel density estimation of all the deconvoluted spectra revealed two primary clusters of peaks, or conformations and a third lowly populated cluster (Fig. 2t). In general, ligands known to be efficacious or strong agonists (e.g., GW1929, rosiglitazone, pioglitazone, and troglitazone) populate the most upfield (right) group of the narrowest peaks in cluster 1. Ligands of other pharmacological types, including partial agonists and inverse agonists, are found in cluster 2 , which is composed of wider peaks, indicative of ensembles composed of multiple conformations. A third lowly populated cluster of downfield chemical shifts and relatively narrow peak widths, cluster 3, occurs for T0070907 and GW9662 ligands that covalently bind to
C313 in the canonical ligand-binding pocket and function as inverse agonists in cell-based assays ${ }^{29}, 30$. These covalent ligands do not attach to the introduced cysteine on helix 12 (C502; Supplementary Fig. 5a, b). PPAR $\gamma^{\mathrm{C} 313 \mathrm{~A}, \mathrm{~K} 502 \mathrm{C}}$-BTFA loaded with or without the same 16 ligands show very similar ${ }^{19} \mathrm{~F}$ NMR spectra to PPAR $\gamma^{\mathrm{K} 502 \mathrm{C}}$-BTFA, except for ligands which covalently bind to $\mathrm{C} 313$, which as expected look similar to ligand-free protein/apoprotein because PPAR $\gamma^{\mathrm{C} 313 \mathrm{~A}, \mathrm{~K} 502 \mathrm{C}}$-BTFA lacks $\mathrm{C} 313$ (Supplementary Fig. 8). Overall, these data indicate a diverse ligand-dependent helix 12 conformational ensemble.

A contribution to the ligand-dependent differences in ${ }^{19} \mathrm{~F}$ NMR chemical shift values could be the relative solvent exposure of the BTFA probe $-\mathrm{CF}_{3}$ group, which can be determined by collecting ${ }^{19} \mathrm{~F}$ NMR spectra as a function of deuterium oxide $\left(\mathrm{D}_{2} \mathrm{O}\right)$ concentration. When increasing amounts of $\mathrm{D}_{2} \mathrm{O}$ are added to a sample, a large upfield shift (i.e., to the right) of the ${ }^{19} \mathrm{~F}$ NMR peak indicates high solvent exposure of the BTFA probe, while a smaller shift indicates low solvent exposure ${ }^{26}$, for example, a solvent-exposed 5-fluorotryptophan shifted 0.217 ppm upfield in $90 \% \mathrm{D}_{2} \mathrm{O}$ buffer ${ }^{33}$. The chemical shift of free BTFA, which has high solvent exposure, has nearly the largest chemical shift change of any measured here ( $0.115 \mathrm{ppm}$ upfield), whereas the solvent-protected MRL24 ligand $\mathrm{CF}_{3}$ group, which is buried deep in the ligand-binding pocket, moves in the opposite direction with increasing $\mathrm{D}_{2} \mathrm{O}$ concentrations (Fig. 3 and Supplementary Fig. 13). Given the solvent-protected position of MRL24 $\mathrm{CF}_{3}$ in the ligand-binding pocket ${ }^{34}$, the movement in the opposite direction of the MRL24 ligand $\mathrm{CF}_{3}$ group likely indicates interaction between the ligand $\mathrm{CF}_{3}$ group and the protein that is

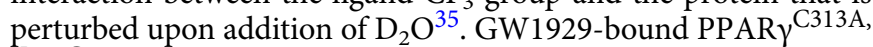
$\mathrm{K} 502 \mathrm{C}$-BTFA has the largest upfield chemical shift $(0.116 \mathrm{ppm}$; Fig. 3), which is consistent with molecular simulations that show a

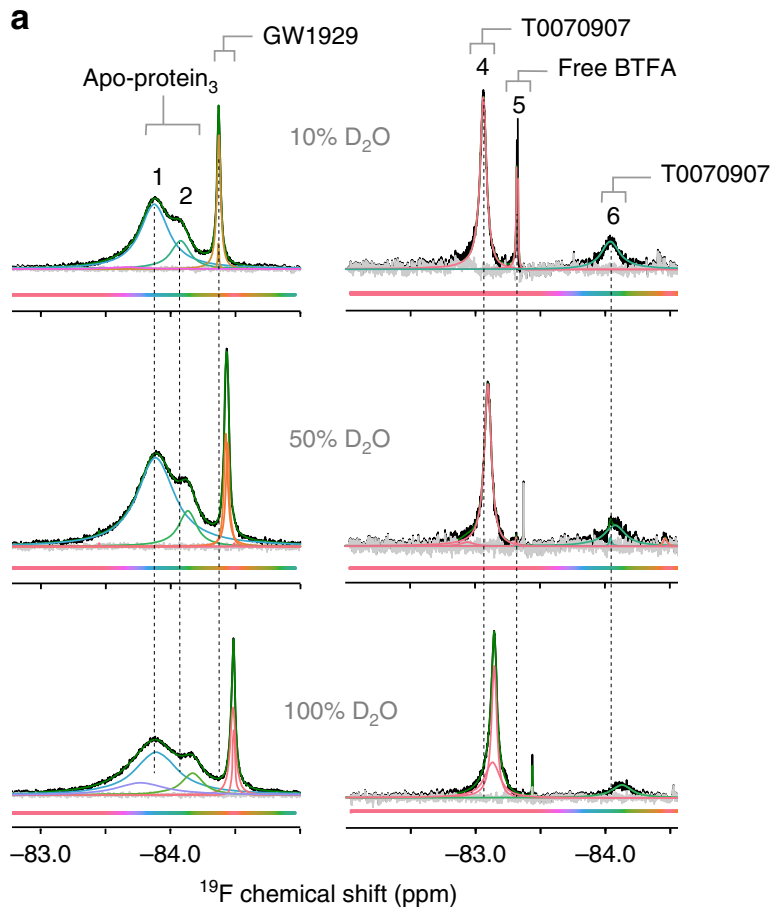

b

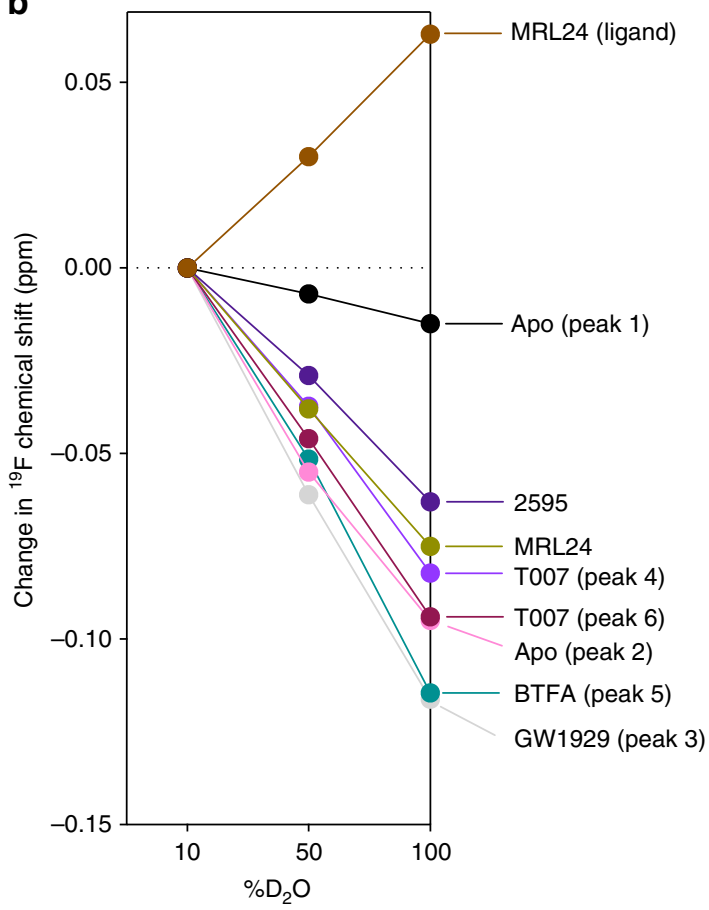

Fig. 3 Helix 12 solvent exposure is distinct for the active and inactive helix 12 conformations. a ${ }^{19} \mathrm{~F}$ NMR spectra of delipidated apo PPAR $\gamma$ C313A,K502C-BTFA bound to 0.2 molar equivalent of GW1929 (left), and T0070907-bound PPAR ${ }^{K 502 C}$-BTFA with excess free BTFA, at the indicated concentrations of $\mathrm{D}_{2} \mathrm{O}$. Due to the high binding affinity of GW1929 (4 nM), all three expected peaks (two apoprotein peaks and one GW1929 peak) are present in slow exchange on the NMR time scale, allowing analysis of the three conformations simultaneously. $\mathbf{b} \mathrm{D}_{2} \mathrm{O}$ solvent isotope-induced changes, plotted as $\% \mathrm{D}_{2} \mathrm{O}$ vs. change ${ }^{19} \mathrm{~F}$ NMR chemical shift values for various peaks; a dotted gray line is shown to highlight no $\mathrm{D}_{2} \mathrm{O}$-induced change in ${ }^{19} \mathrm{~F}$ NMR chemical shift. These experiments were performed once 
a
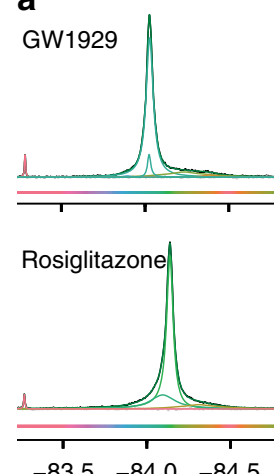

$-83.5-84.0-84.5$
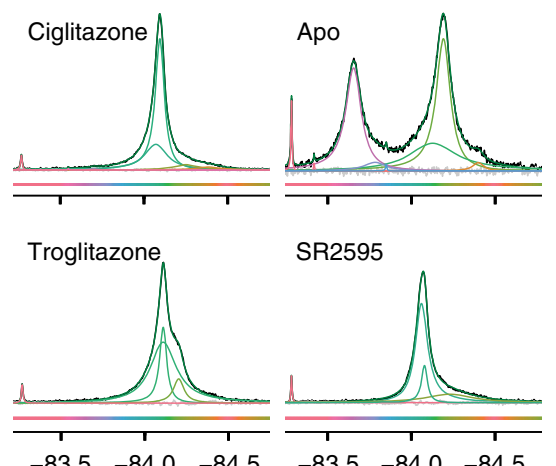

$-83.5-84.0-84.5$

${ }^{19} \mathrm{~F}$ chemical shift (ppm)

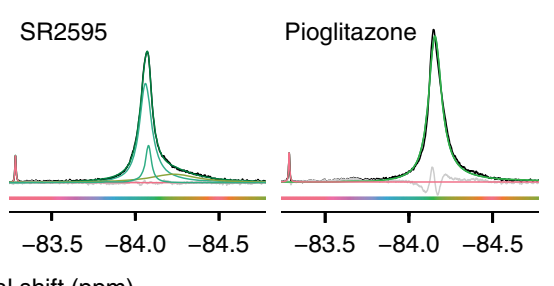

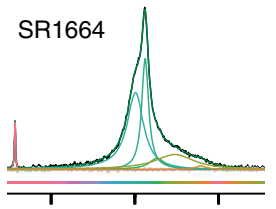

b

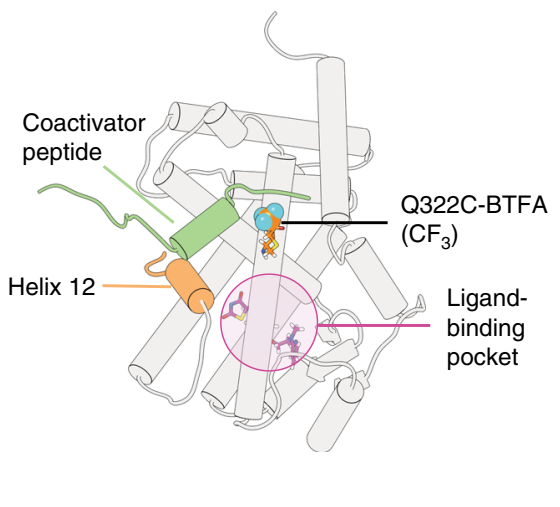

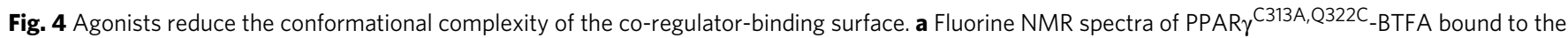

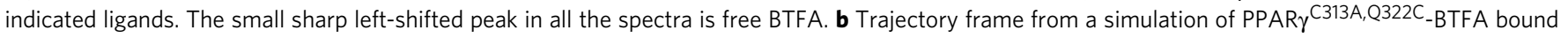

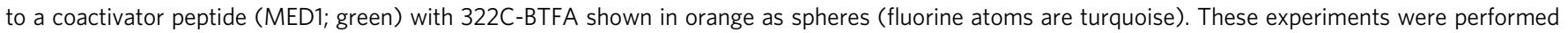
once

that K502 and C502 both have considerable solvent exposure in the active conformation (Supplementary Fig. 3). These data indicate that full agonists primarily populate the active crystalized conformation in solution, while apo, partial agonists, and inverse agonists are found in other distinct conformations.

PPAR $\gamma$ protein expressed in bacteria can pull down mediumchain fatty acids, which function as weak partial agonists ${ }^{31}$. In agreement, non-delipidated apoprotein that is partially bound to endogenous Escherichia coli lipids (Fig. 2s) afforded ${ }^{19} \mathrm{~F}$ NMR spectra with two peaks of similar chemical shift to delipidated apoprotein (Fig. 2r) along with several other lowly populated peaks. Comparison of 2D NMR spectra of delipidated and nondelipidated PPAR $\gamma$ LBD and PPAR $\gamma^{\mathrm{C} 313 \mathrm{~A}, \mathrm{~K} 502 \mathrm{C}_{-} \mathrm{BTFA} \text { in apo }}$ form or bound to ligands indicate that $E$. coli lipids have relatively minor effects on helix 12 and backbone structure (Supplementary Fig. 9a, b). In addition, TR-FRET data collected using nondelipidated or delipidated PPAR $\gamma$-LBD shows essentially the same ligand-dependent co-regulator recruitment potency and efficacy (Supplementary Fig. 9c, d). In separate protein preparations we did observe some variation in T0070907-bound spectra (Supplementary Fig. 10c), which could be due to variable amounts of residual co-bound lipids remaining after delipidation as PPAR $\gamma$ has a very large ligand-binding pocket that can accommodate more than one bound ligand ${ }^{18}$. Overall, these data indicate that some inverse agonists do more than simply displace activating lipids, but instead induce a distinct PPAR $\gamma$ LBD state with higher affinity for corepressors than delipidated apoprotein.

We probed ligand-induced changes in another region of the PPAR $\gamma$ co-regulator-binding surface using a Q322C mutation, which is located in helix 3, generating PPAR $\gamma^{\mathrm{C} 313 \mathrm{~A}, \mathrm{Q} 322 \mathrm{C}}-\mathrm{BTFA}$. The fluorine probe in this variant is solvent exposed in the active conformation and maintains wt-like recruitment of co-regulators (Fig. 4 and Supplementary Fig. 11). The chemical shift difference induced by ligands is relatively small; however, just as with the helix 12 probe, ligands decrease the conformational diversity of this region with the strongest agonists (rosiglitazone and GW1929), yielding the narrowest peaks indicative of a single main conformational ensemble. Similar to apo PPAR $\gamma^{\mathrm{K} 502 \mathrm{C}_{-}}$ BTFA (Fig. 2r), apo PPAR $\gamma^{\mathrm{C} 313 \mathrm{~A}, \mathrm{Q} 322 \mathrm{C}}$-BTFA produces a spectrum with two main wide peaks, indicative of two subensembles composed of multiple conformations with slow exchange between the sub-ensembles (Fig. 4). These data indicate that the ligand-free co-regulator-binding surface is composed of two main structurally diverse (i.e., wide NMR peak) ensembles in slow exchange. Alternatively, one of the peaks could be PPAR $\gamma$ bound to residual $E$. coli lipids; however, this is unlikely given that the apo spectrum is stable over time (Supplementary Fig. 11), whereas E. coli lipid-bound PPAR $\gamma^{\mathrm{K} 502 \mathrm{C}}$-BTFA spectra change with time (Supplementary Fig. 10a). The exchange rate between these two apo NMR peaks is discussed below.

Slow exchange between ensemble structures. A single wellpopulated ${ }^{19} \mathrm{~F}$ NMR peak is observed for BTFA probes placed in two areas of the co-regulator-binding surface (helices 3 and 12) when PPAR $\gamma$-BTFA is bound to a strong agonist such as GW1929 or rosiglitazone. However, for less efficacious ligands, multiple-well-populated ${ }^{19} \mathrm{~F}$ NMR peaks are observed in slow exchange on the NMR time scale indicating that the peaks represent distinct co-regulator-binding surface conformations with lifetimes on the order of milliseconds or longer.

To confirm the apparent slow exchange between different conformations, we performed ${ }^{19} \mathrm{~F}$ chemical exchange saturation transfer NMR experiments on PPAR $\gamma$ where multiple peaks, or conformations, are present (Fig. 5a and Supplementary Fig. 12). Of the five ligands and apoprotein studied, four ligands and apoprotein show obvious slow chemical exchange between a wellpopulated minor resonance and the most abundant resonance. In the case of T0070907, exchange was difficult to detect, likely because exchange rates $<\sim 0.4 \mathrm{~s}^{-1}$ are too slow to be effectively detected using this method under our experimental conditions (Supplementary Fig. 12a, b).

We next quantified the exchange rate between peaks. PPAR $\gamma^{\text {K502C }}$-BTFA bound to GW9662 and troglitazone each showed very slow exchange between peaks, making precise measurement difficult (0.3 and $0.4 \mathrm{~s}^{-1}$; Supplementary Fig. 12d). However, for pioglitazone and ciglitazone, the exchange between the two prominent peaks is 1.2 and $1.4 \mathrm{~s}^{-1}$ (95\% confidence interval (CI): $1.1-1.3 \mathrm{~s}^{-1}$ for pioglitazone and $1.3-1.5 \mathrm{~s}^{-1}$ for ciglitazone; Fig. 5b). In addition, we found exchange of $1.0 \mathrm{~s}^{-1}$ (95\% CI: $0.9-1.1 \mathrm{~s}^{-1}$ ) between the two resolved apo peaks of PPAR $\gamma^{C 313 A, Q 322 C}$-BTFA (Supplementary Fig. 12c). These slow exchange rates are consistent with the notion that the detected conformations originate from larger scale movements involving many atoms ${ }^{36}$, indicating helix 12 and the co-regulator-binding surface exchanges between two or more distinct conformations. Almost all of the signal in the ligand-bound spectra do not overlap with the apo spectrum, thus implying that exchange between conformations is occurring while bound to ligand. These data raise the possibility that these ligands are found in at least 

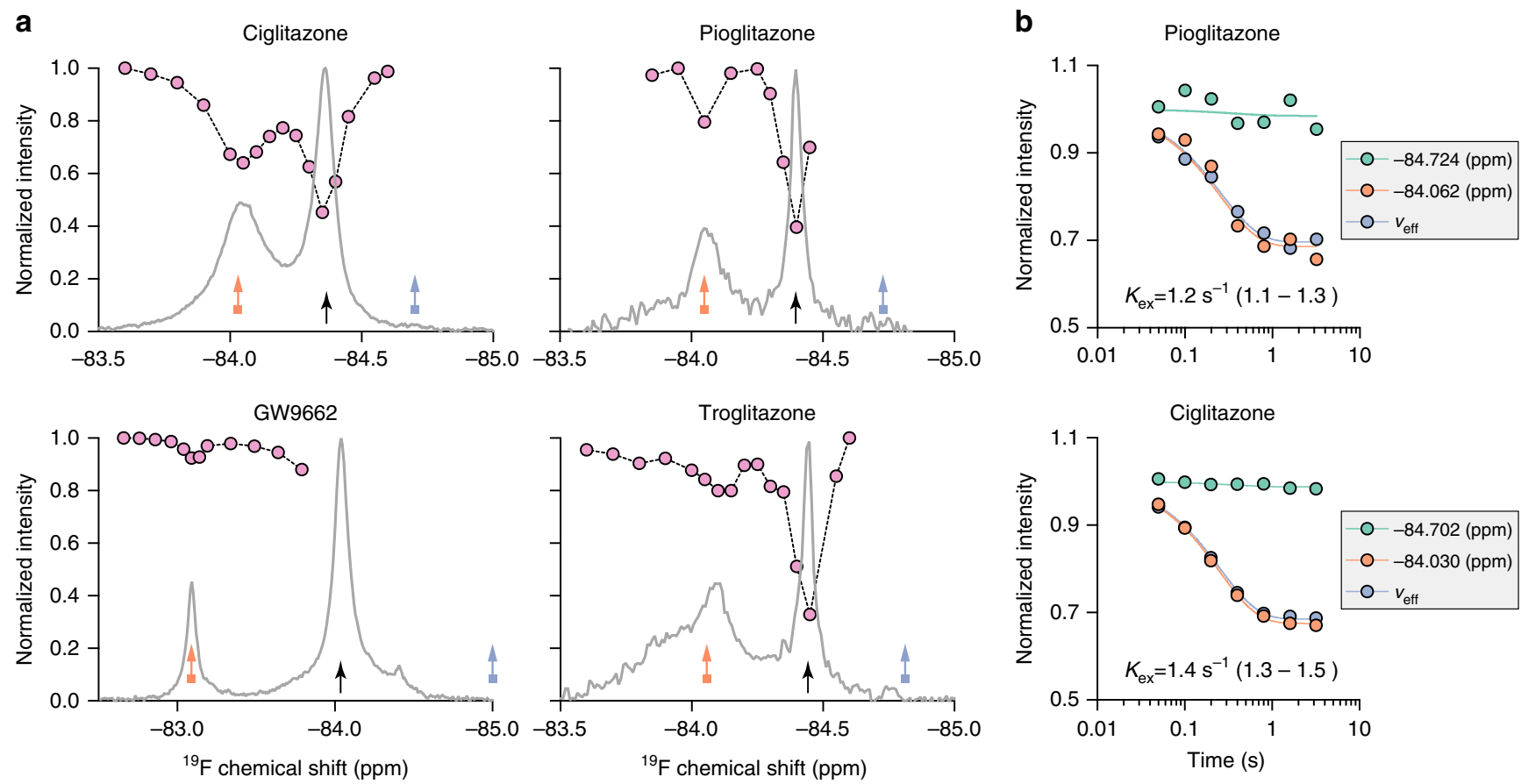

Fig. 5 Chemical exchange saturation transfer indicates slow exchange between multiple helix 12 conformations. a A selective Gaussian pulse was used to saturate the ${ }^{19} \mathrm{~F}$ spectra bound to the indicated ligands at locations indicated by pink circles. The height of the pink circles indicates the height of the peak indicated by the black arrow when the selective pulse was carried out at the chemical shift of the pink circle. If exchange is occurring between the two peaks, the pink dots should mirror the spectrum. b A selective Gaussian pulse was used to saturate the spectrum at an on resonance (a; orange box arrow) and off resonance (a; blue box arrow) location, the peak height of the most abundant resonance (a; black arrow) was monitored as a function of the duration of the saturation pulse, and the resulting peak intensities were fit to extract the exchange rate. Ninety-five percent confidence intervals for the fit

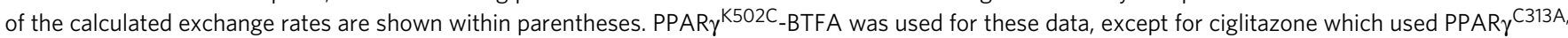
K502C -BTFA for increased signal to noise. These experiments were performed once

two functionally distinct main conformations exchanging on the seconds time scale. This idea is consistent with the fact that the PPAR $\gamma$ non-agonist/antagonist SR1664 has been crystalized in two distinct conformations ${ }^{13,37}$.

Connecting the helix 12 structural ensemble to function. To test whether the ${ }^{19} \mathrm{~F}$ NMR detected helix 12 conformations correlate to function within our set of 16 ligands, we compared the mean-weighted ${ }^{19} \mathrm{~F}$ NMR chemical shift values to ligand efficacy for recruitment of MED1 (coactivator), NCoR (corepressor), and for a subset of these ligands, CBP (coactivator) in the TR-FRET co-regulator interaction assay. For PPAR $\gamma^{\mathrm{K} 502 \mathrm{C}}$-BTFA there is a correlation between mean ligand-induced ${ }^{19} \mathrm{~F}$ NMR chemical shift and ligand-induced MED1, CBP, and NCoR recruitment efficacy (Fig. 6a). There are similar correlations for PPAR $\gamma^{\mathrm{C} 313 \mathrm{~A} \text {, }}$

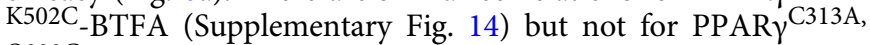
Q322C_BTFA. In addition, we used FP to measure the affinity of a subset of PPAR $\gamma$-ligand complexes for co-regulators (MED1, NCoR, and SMRT) and compared these to labeled and wt PPAR $\gamma$ and found that ligand-specific affinity correlates with NMR chemical shift (Fig. 6b and Supplementary Fig. 14). The most efficacious agonist for MED1 and CBP binding in our ligand set (GW1929) induces the most upfield mean chemical shift

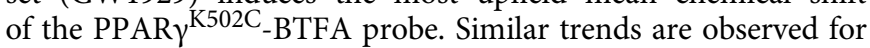
other agonists, including the right-shifted peaks of thiazolidinedione (TZD/glitazones) ligands. In contrast, downfield mean chemical shifts are prevalent in apoprotein and inverse-agonist (T0070907)-bound PPAR $\gamma^{\text {K502C }}$-BTFA, which show the highest efficacy and affinity for NCoR and SMRT binding (Fig. 6). These data indicate that the conformations detected by ${ }^{19} \mathrm{~F}$ NMR are functionally distinct.
Co-regulators shift the conformational ensemble. The data above demonstrate that ligands of different pharmacological activities can stabilize functionally distinct co-regulator-binding surface conformations. To determine how co-regulator-binding influences these ligand-dependent conformational ensembles in solution, we performed ${ }^{19} \mathrm{~F}$ NMR with and without coactivator (MED1 and CBP) and correpressor (SMRT and NCoR) peptides, and in the absence or presence of the most efficacious agonist (GW1929) or inverse agonist (T0070907) and a less efficacious inverse agonist/antagonist (GW9662) (Fig. 7a). Similar to strong agonists, addition of coactivators to apoprotein induces an upfield (right) shifting of the spectra. In contrast, similar to inverse agonists, addition of corepressors to apoprotein induces a downfield (left) shifting of the spectrum. Notably, cor-

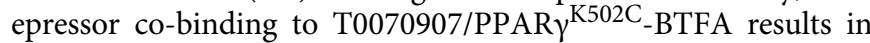
smaller perturbations to the fluorine spectra than coactivator cobinding. The opposite is observed for co-regulator co-binding to GW1929/ PPARy ${ }_{\text {K502C }}$ BTFA. Apoprotein-bound and GW9662bound PPAR $\gamma^{\mathrm{K} 502 \mathrm{C}}$-BTFA are almost equally changed by the addition of coactivators or corepressors (Fig. 7c). These data suggest that the GW1929/PPAR $\gamma$ and T0070907/PPAR $\gamma$ coregulator-binding surface structural ensembles are near ideal for MED1/CBP and NCoR//SMRT binding, respectively. In contrast, the apoprotein and GW9662 structural ensembles are not ideal for binding any of these co-regulators.

RXRa heterodimerization shifts the inverse agonist ensemble. PPAR $\gamma$ binds enhancer regions on DNA and affects gene expression primarily as a heterodimer with $\mathrm{RXRa}^{38}$. We therefore performed experiments to determine the effect of RXRa heterodimerization on the conformational ensemble and 
a
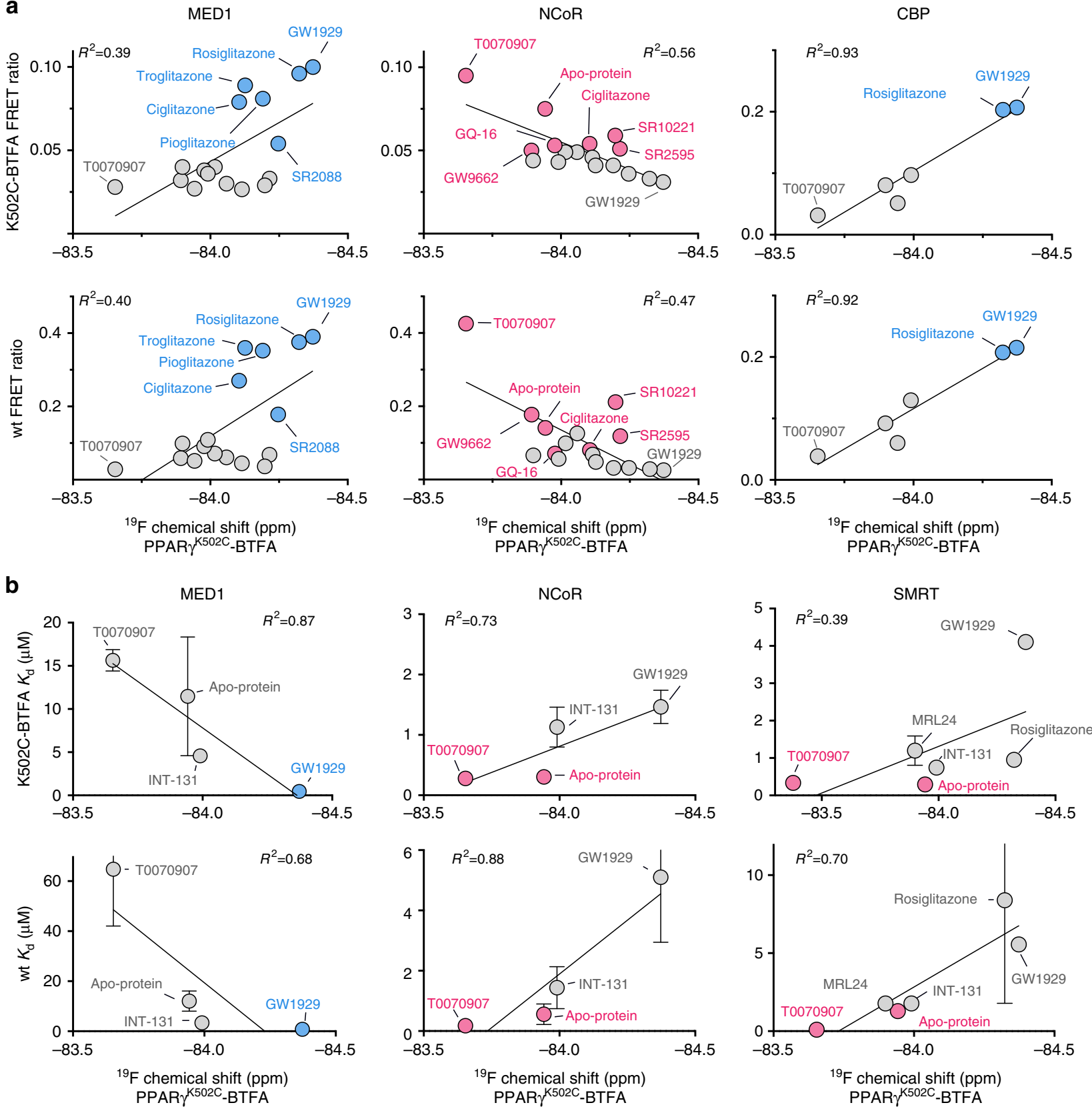

Fig. 6 Ligand-directed helix 12 ensemble dictates PPAR $\gamma$-co-regulator interaction. a Plot of mean ${ }^{19} \mathrm{~F}$ NMR chemical shift values (PPAR ${ }^{\mathrm{K} 502 \mathrm{C}}$-BTFA) vs. TR-FRET endpoint data for the recruitment of MED1, NCoR, and CBP peptides to PPAR ${ }^{\text {K502C }}$-BTFA (top panels) and wt PPAR LBD (bottom panels) for the set of 16 pharmacologically distinct synthetic PPAR ligands and apoprotein (select ligands for CBP). Error bars are relatively small for endpoint TRFRET values (Fig. 1 and Supplementary Figure 6) and are excluded for clarity. b Plot of mean ${ }^{19}$ F NMR chemical shift values (PPAR ${ }^{\text {K502C }}$-BTFA) vs. MED1, $\mathrm{NCoR}$, and SMRT peptide dissociation constant $\left(K_{d}\right)$ for PPAR $\gamma^{\mathrm{K} 502 \mathrm{C}}$-BTFA (top panels) and wt PPAR $\mathrm{LBD}$ (bottom panels) as measured by fluorescence polarization for a subset of the ligands in $\mathbf{a}$. Linear regression fit is shown as a solid line and the correlation coefficient $\left(R^{2}\right)$ for the fitted line is indicated. The ligands with the highest efficacy for MED1 and NCoR recruitment for PPAR ${ }^{\mathrm{K} 502 \mathrm{C}}$-BTFA are highlighted. Error bars represent standard deviation of two (K502C-BTFA $K_{\mathrm{d}}$ and wt SMRT $K_{\mathrm{d}}$ ) or three (wt MED1 and NCoR $K_{\mathrm{d}}$ ) independent experiments

on co-regulator recruitment. Other than the expected broadening and consequently decreased signal from the larger molecular weight complex, RXRa heterodimerization has a relatively small effect on the ${ }^{19} \mathrm{~F}$ spectra of PPAR $\gamma^{\mathrm{C} 313 \mathrm{~A}, \mathrm{~K} 502 \mathrm{C}}$-BTFA when cobound to GW1929 (agonist), MRL24 (less efficacious partial agonist), and apo. RXRa binding to PPAR $\gamma^{\mathrm{K} 502 \mathrm{C}_{-} \mathrm{BTFA}}$ cobound to T0070907 (inverse agonist) induces a large change in the spectrum, decreasing the relative population found in the leftshifted peak (Fig. 8a), which is expected to disfavor NCoR binding. Consistent with the change in ${ }^{19} \mathrm{~F}$ NMR spectrum,
NCoR binding is decreased and MED1 recruitment is increased by RXR $\alpha$ heterodimerization with the T0070907/PPAR $\gamma$ complex (Fig. 8).

Simulations suggest a diverse ligand-dependent ensemble. The extreme broadening observed in apo PPAR $\gamma$ alone or bound to NCoR peptide originate from multiple conformations exchanging every $\mu$ s to ms. We reasoned that we could sample some of these conformations in independent long time-scale simulations of apo 
a

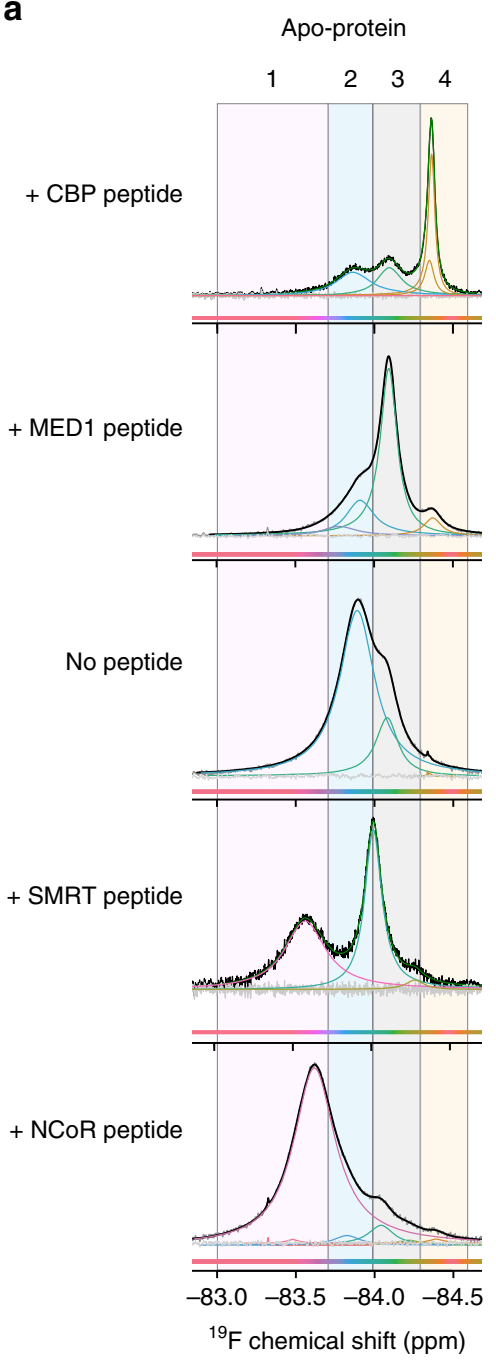

GW1929

(Strong MED1 binding)
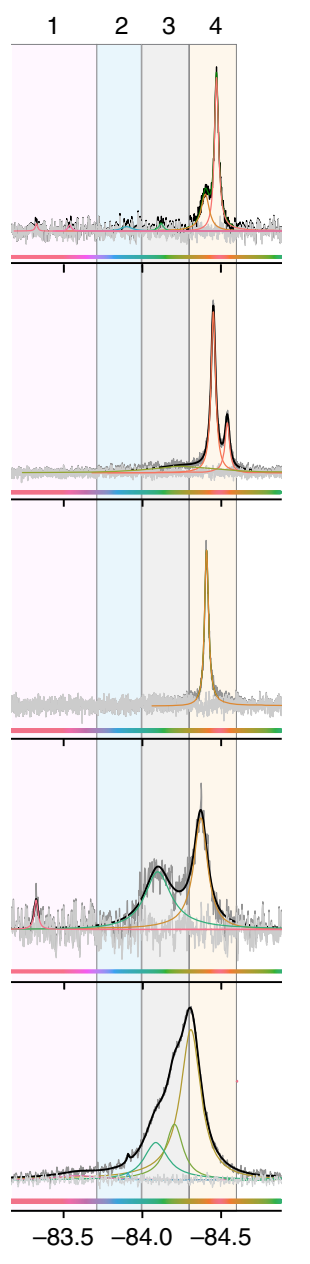

${ }^{19} \mathrm{~F}$ chemical shift (ppm)

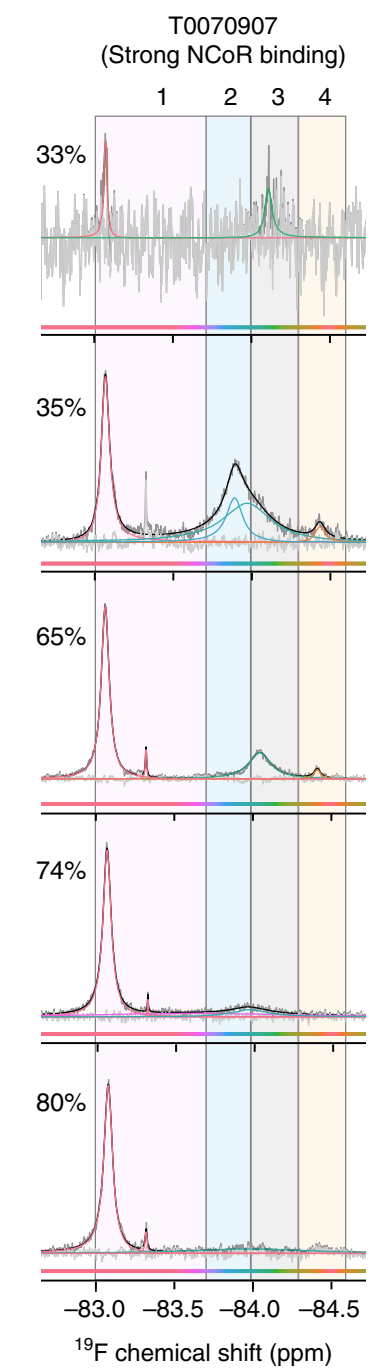

b

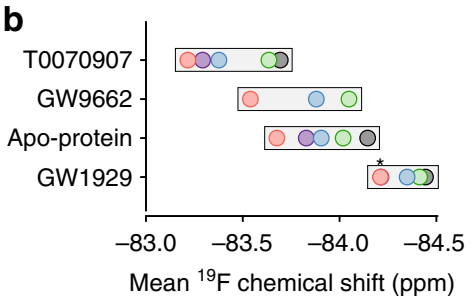

C

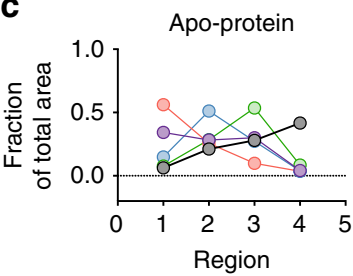

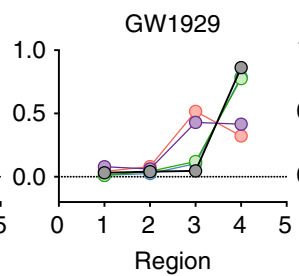

Region

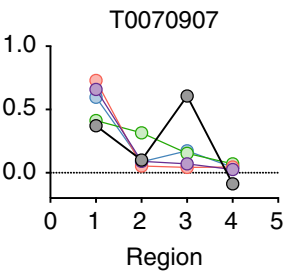

Region
GW9662

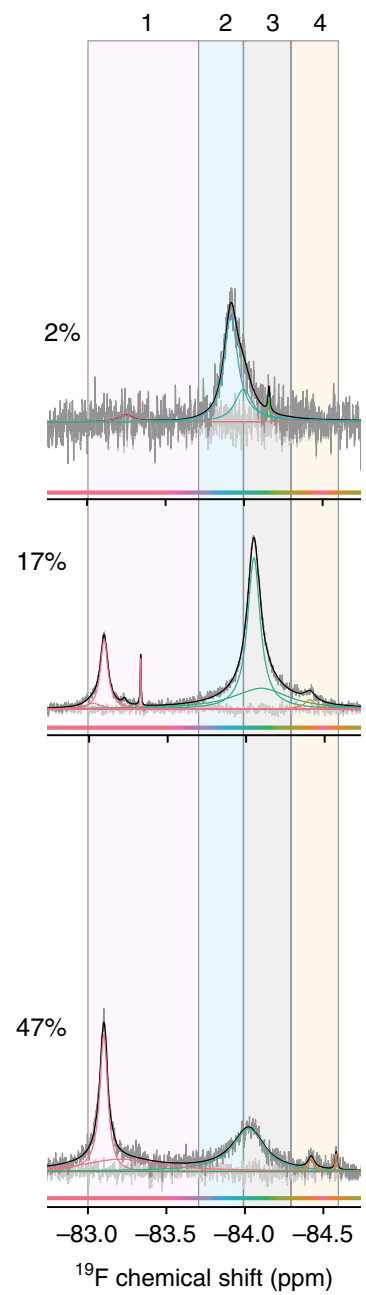

$$
\square \text { NCoR } \square \text { SMRT } \square \text { No peptide } \square \text { MED1 } \square \text { CBP }
$$

Fig. 7 Co-regulator-binding shifts the helix 12 conformational ensemble. a Deconvoluted ${ }^{19} \mathrm{~F}$ NMR spectra of apo PPAR $\gamma{ }^{\mathrm{C} 313 \mathrm{~A}, \mathrm{~K} 502 \mathrm{C}-\mathrm{BTFA} \text { and }}$ PPAR $\gamma^{\text {K502C }}$-BTFA bound to GW1929 or T0070907 in the absence and presence of MED1 coactivator or NCoR corepressor peptides. The percent of total signal area found in the left sharp peak is shown for T0070907 and GW9662 spectra. The small sharp peak at -83.3 ppm is free BTFA. b Mean-weighted chemical shift values from the plots in a. c Fraction of the total peak areas in the four colored boxed regions in a, which roughly correspond to the clustered spectral regions from Fig. 2; with the two middle regions corresponding to the two peaks observed for apoprotein. Agonist-bound PPAR $\gamma$ is changed little

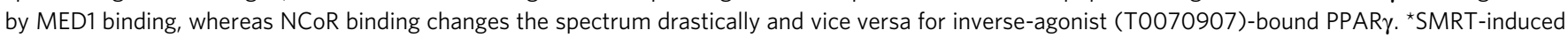
mean chemical shift in GW1929-bound PPAR $\gamma^{\text {K502C }}$-BTFA is the same as NCoR. These experiments were performed once

alone or apo bound to NCoR. We also simulated inverse agonist (T0070907)-bound PPAR $\gamma$ LBD with and without co-bound NCoR to gain additional insight into how this ligand changes the co-regulator-binding surface conformational ensemble. We built simulation models using crystal structures with helix 12 in an inactive chain B conformation (Supplementary Fig. 2d) to avoid steric clash with bound NCoR peptide. We ran simulations at $37^{\circ}$ $\mathrm{C}$ (NMR was run at $25^{\circ} \mathrm{C}$ ) and with the TIP3P water model
(TIP3P is much less viscous than actual water ${ }^{39}$ ) to speed relaxation to a local or global energy minimum (i.e., stable conformation) given that this chain B-inactive conformation may be in a higher energy conformation. These simulations were allowed to run until reaching a structure that remained reasonably stable for at least $5 \mu \mathrm{s}$ as judged by consistent helix 12 RMSD relative to the starting structure (Supplementary Fig. 15). These stable conformations are representative of a local or global free 

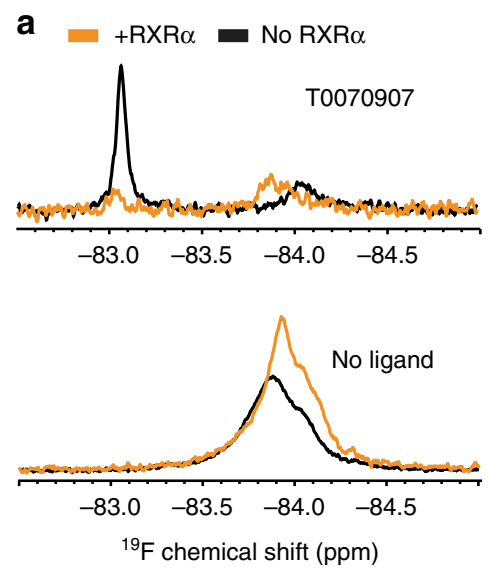

b

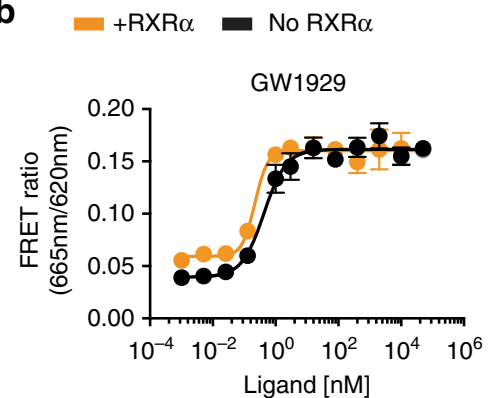

C

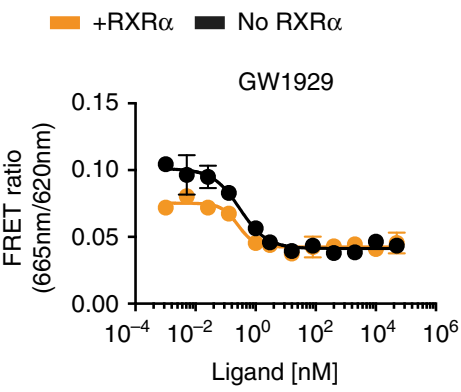

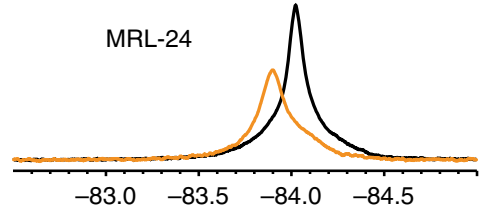
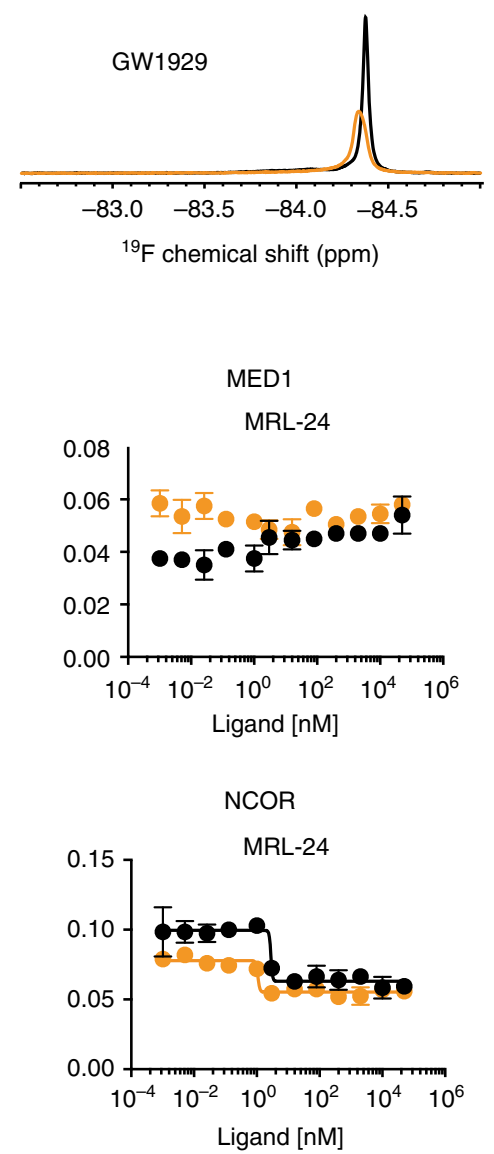

d

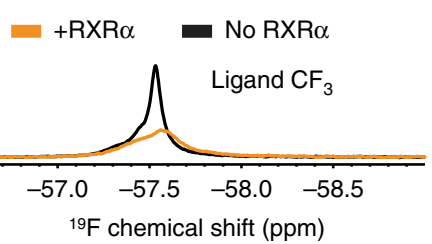

e

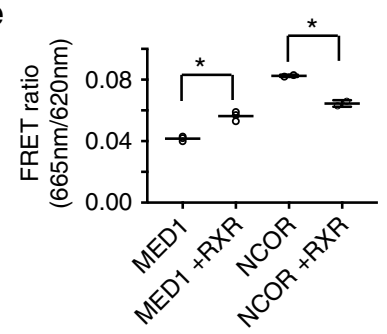

T0070907
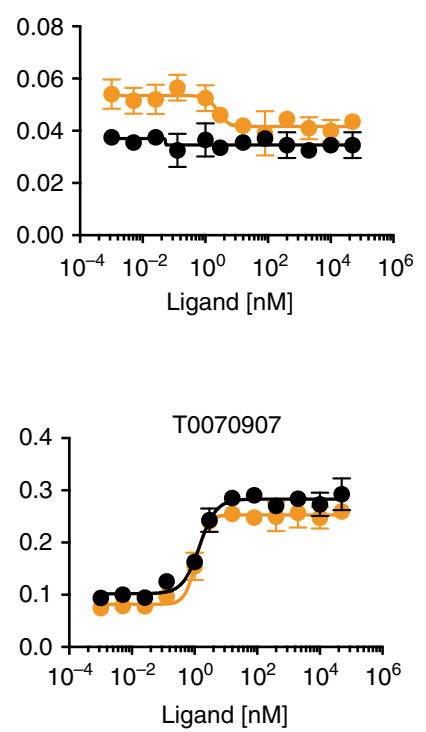

Fig. 8 Heterodimerization of PPAR $\gamma$ LBD with RXR $\alpha$ LBD favors coactivator binding. a ${ }^{19}$ F NMR of PPAR ${ }^{K 502 C}$-BTFA bound to T0070907 and PPAR $\gamma$ C313A,502C - BTFA bound to MRL24 or GW1929 or with no ligand bound was performed in the presence (orange) or absence (black) of RXR $\alpha$ LBD. These experiments were performed once. $\mathbf{d}$ The ${ }^{19} \mathrm{~F}$ NMR signal from the MRL24 ligand. Broadening and consequent reduction in signal intensity is expected as a consequence of the increased rotational correlation time of the heterodimer complex. $\mathbf{b}, \mathbf{c}$ TR-FRET was used to measure interaction between wt PPAR $\gamma$ LBD and MED1 or NCoR in the presence or absence of equimolar concentrations of RXR $\alpha$. Error bars represent standard deviation of two technical replicates within a single experiment. The experiment was repeated twice and gave similar results each time. e Heterodimerization favors MED1 binding $(p=0.0017)$ and disfavors NCoR binding $(p=0.0076)$ to apo PPAR $\gamma$. In addition, visual and statistical comparison of NCoR and MED1 recruitment to PPAR LBD saturated with ligand (four highest concentrations of ligands) indicates that RXR $\alpha$ affects co-regulator recruitment to T0070907-bound PPAR $(N C o R, p=0.0042 ;$ MED1, $p=0.0027$ ) more than GW1929 (NCoR, $p=0.44 ;$ MED1, $p=0.34)$ or MRL24 (NCoR, $p=0.0141 ;$ MED1, $p=0.061)$. All $p$ values are derived from a two-tailed $t$ test

energy minima. Three of four independent simulations of PPAR $\gamma$ LBD alone (apo) relaxed to a cluster of distinct but similar conformations that are somewhat similar to the starting chain $\mathrm{B}$ crystal structure, while the fourth does not appear to stabilize to the same degree and goes to a distinct conformation. These results are consistent with the broad ligand-free (apo) PPAR $\gamma{ }^{19} \mathrm{~F}$ peaks observed which indicate exchange between two or more conformations (Fig. 9a) and are also consistent with previous free energy calculations that indicated that the inactive chain $B$ crystal structure is very similar to low-energy apo structures ${ }^{40}$. Addition of NCoR to apo PPAR $\gamma$ LBD results in up to $12 \mu \mathrm{s}$ of relative instability (Supplementary Fig. 15b) before finally relaxing to several different stable conformations that are consistent with the idea that NCoR pushes helix 12 away from the co-regulator-binding surface, yielding a diverse collection of conformations that are consistent with the observed broad helix $12{ }^{19}$ F NMR peaks observed for this complex (Fig. 9b). All three simulations of T0070907 bound to PPAR $\gamma$ LBD relax to very distinct conformations (Fig. 9c). Addition of NCoR to T0070907bound PPAR $\gamma$ provides a path to one dominant energy minima as all three simulations in this complex reach conformations similar to an active conformation, but with helix 12 shifted to accommodate the longer LXX I/H IXXX I/L helix of corepressors compared to the LXXLL motif of coactivators ${ }^{41}$. These data are consistent with our ${ }^{19} \mathrm{~F}$ NMR data that demonstrate that NCoR binding to the T0070907 PPAR $\gamma$ LBD complex induces a shift towards one particular conformation from several conformations (Fig. 9d). 
a
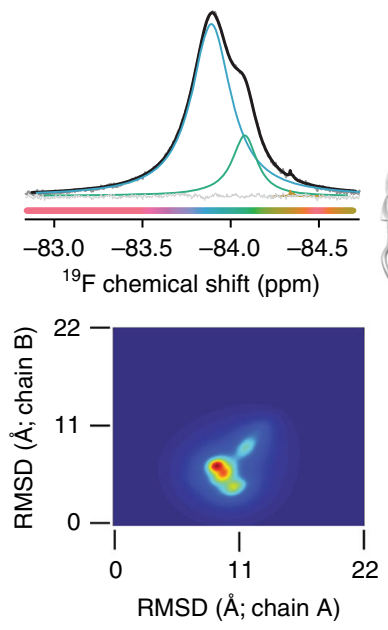

C
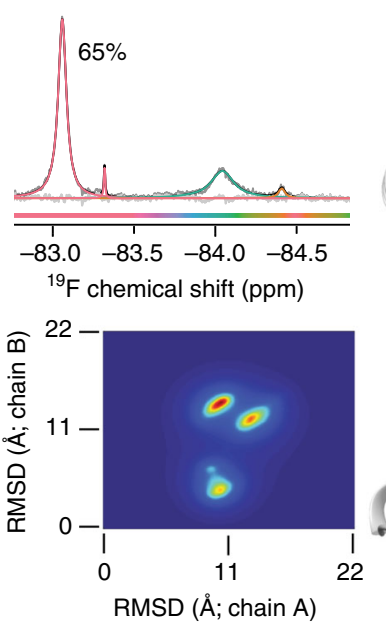

e

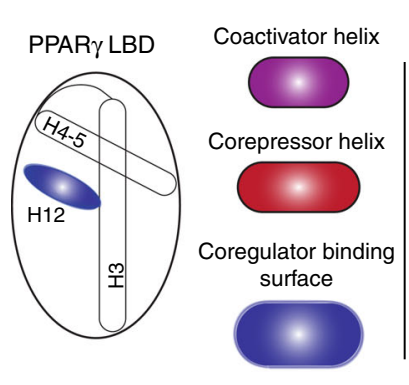

Apo

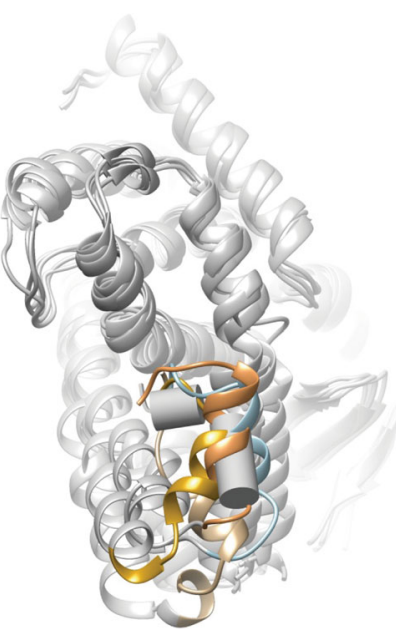

nverse agonist

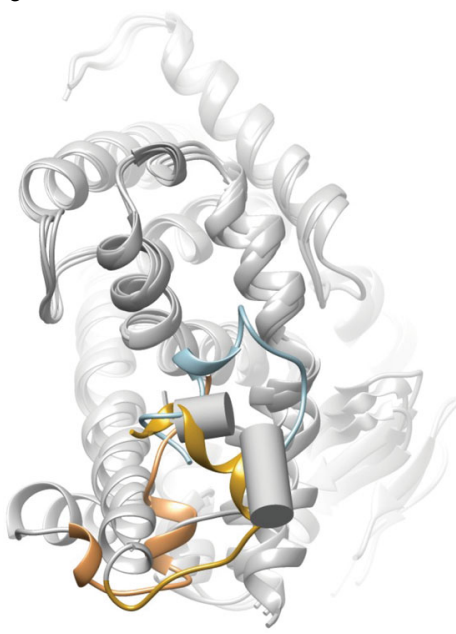

b

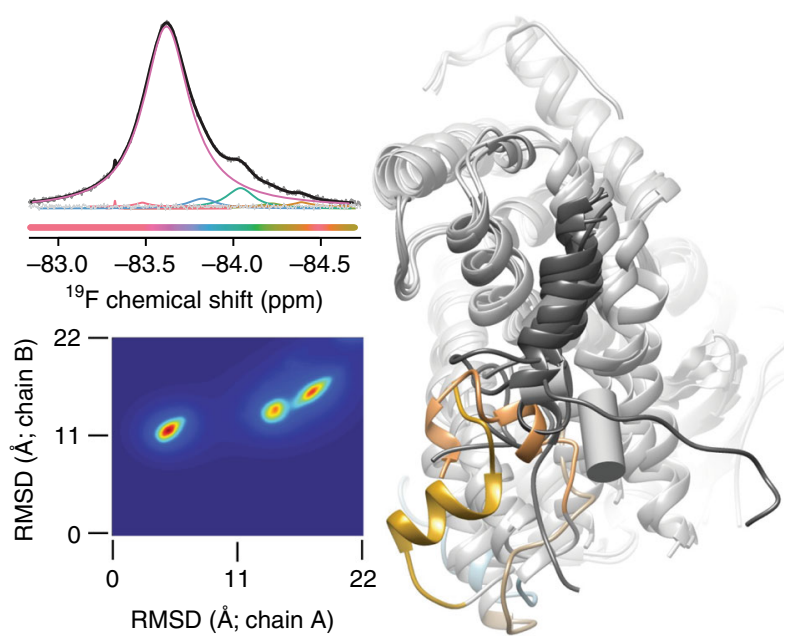

d
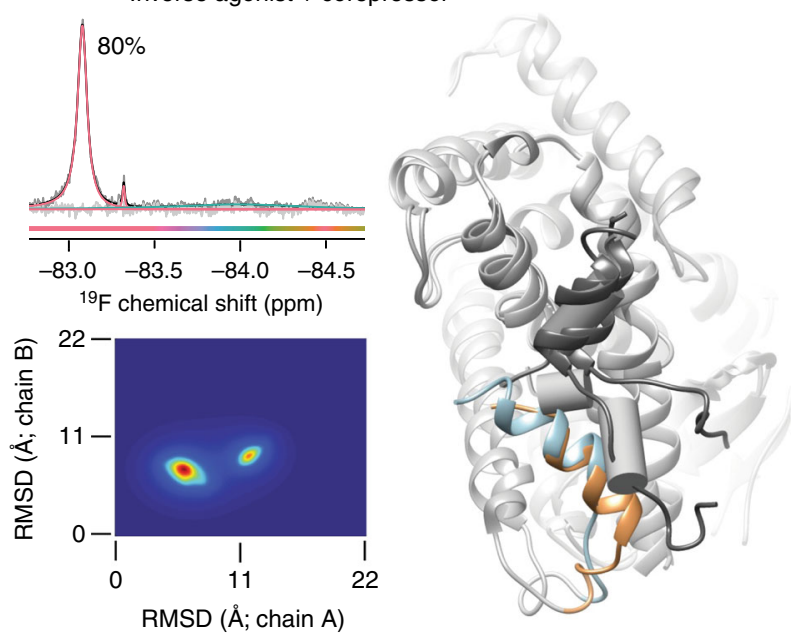

Apo + Agonist $\quad+$ Agonist \& coactivator Apo/+partial agonist

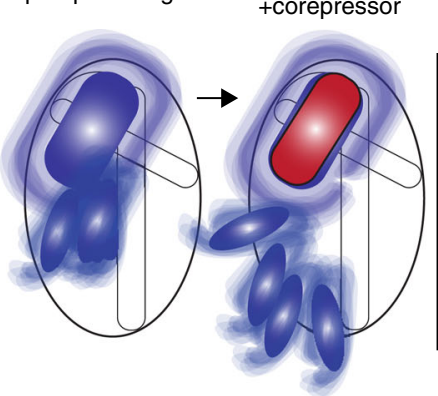

+Inverse agonist + +Inverse agonist \& corepressor

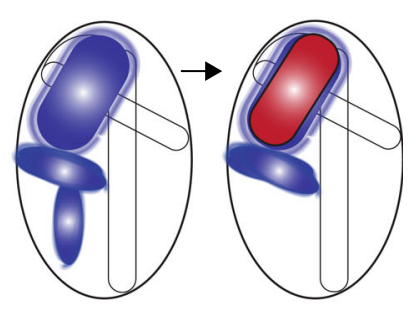

Fig. 9 Microsecond time-scale molecular simulations point to a possible NCoR bound structure. a-d Root mean square deviation (RMSD) of helix 12 relative to an inactive (1PRG chain B) structure and an active conformation (1PRG chain A) for helix 12 in four different simulated complexes. Helix 12 from these inactive and active structures is shown in all panels (gray pipe). SMRT bound to PPAR (PDB code: 1KKQ) was aligned to 1 PRG chain A and the SMRT helix is shown in $\mathbf{b}$ and $\mathbf{d}$ (gray pipe). Fourteen independent simulations of $\mathbf{a}$ apo PPARy LBD, $\mathbf{b}$ apo + NCoR corepressor, $\mathbf{c}$ inverse-agonist (T0070907)-bound PPAR LBD, and $\mathbf{d}$ PPAR $\gamma$ LBD co-bound to T0070907 and NCoR corepressor were carried out for 10 to $30 \mu$ and the last $5 \mu$ s were analyzed (4 each for apo simulations and 3 each for T0070907 simulations). Colors on the RMSD plots indicate relative number of frames; red indicates the most prevalent and blue the least. Representative structures from the centroid of the major clusters are shown with helix 12 highlighted in color (4 apo, 4 apo + NCOR, 3 T0070907 and 2 T0070907 + NCoR). Helix 12 disassociates from the LBD in one apo + NCoR simulation. The cluster for this simulation (centered at $21.3 \AA(x)$ and $23.8 \AA(y))$ is not shown; however, a representative structure is shown in light blue. Corresponding ${ }^{19} \mathrm{~F}$ NMR data from Fig. 7 are shown in each panel. e A model for helix 12 conformational diversity based on simulation and experiment. Apo PPAR or partial-agonist-bound PPAR $\gamma$ helix 12 is found in many similar conformations of varying helical structure producing broad NMR peaks and rapid hydrogen deuterium exchange, while full agonist is found in a tighter cluster of conformations. Co-binding of the inverse agonist T0070907 and the corepressor NCoR produces one main conformation similar to an active conformation, but with helix 12 shifted. Fuzziness implies intermediate exchange ( $\mu$ s to ms) between the conformations 


\section{Discussion}

The data presented here explicitly reveal the conformational ensemble of the co-regulator-binding surface, including helix 12, of a nuclear receptor and the effects of ligands on that ensemble. A model of nuclear receptor activation arises from these data and previously published work, which indicates that nuclear receptors and other proteins are found in ensembles of structures and not a single structure ${ }^{17,22-24,42,43}$. The structural variance around this primary structure varies from protein to protein; however, for nuclear receptors this variance appears to be considerable ${ }^{16,17,44}$. The data presented here indicate that the co-regulator-binding surface, including helix 12 , exchanges relatively quickly (i.e., $\mu$ s to ms lifetimes) between many conformations for apo PPAR $\gamma$ and partial-agonist-bound PPAR $\gamma$. Agonist and inverse agonist binding consolidates this complex apo ensemble into structurally distinct active or inactive state(s) which favor coactivator or corepressor binding respectively (Figs. 7 and 9e). Very slow exchange (seconds) across high kinetic barriers is observed for some agonists and inverse agonists, indicating that these ligands hold helix 12 in narrow and deep energy wells with rare exchange occurring between sub-ensembles (Fig. 5). Consistent with these data, free energy calculations indicate that helix 12 in apo PPAR $\gamma$ is found in two broad energy wells with conformations similar to both active and inactive apo PPAR $\gamma$ helix 12 crystal conformations, whereas helix 12 in rosiglitazone-bound PPAR $\gamma$ is found in one deep narrow well with a conformation similar to the active chain A conformation ${ }^{40}$. Importantly, these data reveal a correlation between ligand efficacy and the prevalence of at least three distinct structural ensembles using a diverse set of 16 pharmacologically distinct PPAR $\gamma$ ligands.

The long time-scale simulations presented here are qualitatively consistent with the experimental results. Simulations started from an inactive chain B structure of PPAR $\gamma$ relax to multiple different structures depending on the ligand and co-regulator that are bound to PPAR $\gamma$ (Fig. 9). Apo PPAR $y$ LBD relaxes to conformations similar to the starting inactive chain $B$ structure, while NCoR binding pushes helix 12 into various different structures, consistent with the idea that NCoR does not interact productively with helix 12 in apo PPAR $\gamma$. In contrast, simulations of NCoR and T0070907 co-bound to PPAR $\gamma$ relax to two very similar structural clusters, which are consistent the idea that helix 12 interacts productively with $\mathrm{NCoR}$ in this complex. In addition, simulations of GW1929 bound to PPAR $\gamma$ starting with helix 12 in an active chain A conformation remain in a similar helix 12 conformation throughout the simulation (Supplementary Fig. 3), indicating that as expected this is the dominant conformation for agonist-bound PPAR $\gamma$ in solution. These simulations also indicate that the length and helicity of helix 12 can weaken in some conformations (Fig. 9), which could contribute to broad ${ }^{19}$ F NMR peaks and increased exchange rates in HDX-MS ${ }^{13}$. Overall, it is encouraging that these non-converged simulations qualitatively agree with ${ }^{19} \mathrm{~F}$ NMR and provide a glimpse of possible conformations that comprise a portion of the observed ${ }^{19} \mathrm{~F}$ NMR spectra; however, quantitative comparison between the ${ }^{19} \mathrm{~F}$ NMR spectra and converged simulations remains a future challenge.

This view of the co-regulator-binding surface, including the number and relative populations of conformations and subensembles that comprise the ensemble is made possible by using a single fluorine probe which provides high sensitivity, a single signal which obviates the need to transfer spin between residues, and a total lack of background signal. ${ }^{19} \mathrm{~F}$ NMR requires mutation and labeling of PPAR $\gamma$ which perturbs corepressor affinity (especially SMRT) and has little effect on coactivator affinity as measured by TR-FRET peptide recruitment and FP affinities for corepressor (NCoR and SMRT) and coactivator (MED1 and CBP) peptides (Supplementary Fig. 7). Based on these observed functional effects, we propose that any effect from the mutations would likely shift inverse-agonist-bound mean NMR chemical shifts toward the center decreasing the population found in the inverse agonist conformational state (cluster 3). Depending on the chemical shift difference and rate of exchange between the conformations this will result in movement of a peaks chemical shift and/or a decrease in population of the cluster 3 (left cluster). For example, a left-shifted narrow peak at a chemical shift typical of inverse agonism (cluster 3 ) and a broad peak at a chemical shift typical of antagonist/partial agonist (cluster 2) are observed for PPAR $\gamma^{\text {K502C }}$-BTFA bound to the inverse agonist T007090729, 30 and less efficacious inverse agonist/antagonist GW9662 (Figs. 2 and 7). Addition of NCoR or SMRT shifts the population from cluster 2 to cluster 3, while the mutations and labeling may shift the equilibrium population in the opposite direction toward the antagonist/partial agonist cluster (cluster 2). Thus, it may be that helix 12 of wt PPAR $\gamma$ LBD bound to T0070907 is found in a conformation represented by the inverse agonist cluster (cluster 3; left-shifted narrower peak) to a larger degree than detected by PPAR $\gamma^{\mathrm{K} 502 \mathrm{C}}$-BTFA.

This work adds detail to how ligands in general control the activity of nuclear receptors. Our data not only confirm that helix 12 and the co-regulator-binding surface exist as a ligand-specific dynamic structural ensemble but also reveal the relative populations of sub-ensembles that comprise the overall structural ensemble and correlate function with this ensemble. Further definition of the conformational ensemble of the entire protein and the kinetics and thermodynamics of exchange between the members of the ensemble will build an accurate model of how ligands produce functional outputs via nuclear receptors and allow greater control of their function via ligands.

\section{Methods}

Protein purification. A pET-46 plasmid carrying the genes for ampicillin resistance and $\mathrm{N}$ terminally 6xHis-tagged PPAR $\gamma$ containing a tobacco etch virus (TEV) nuclear inclusion protease recognition site between the His tag and protein of interest was transformed into chemically competent $E$. coli BL21(DE3) Gold cells (Invitrogen). Cells were grown in either ZYP-5052 autoinduction media or terrific broth (TB). Cells grown in $\mathrm{TB}$ at $37^{\circ} \mathrm{C}$ were induced at an $\mathrm{OD}_{600}$ of approximately 0.8 by the addition of $0.5 \mathrm{mM}$ isopropyl $\beta$-D-1-thiogalactopyranoside (IPTG) and the temperature lowered to $22^{\circ} \mathrm{C}$. Induction proceeded for $16 \mathrm{~h}$ prior to harvesting. Harvested cells were homogenized into $50 \mathrm{mM}$ phosphate ( $\mathrm{pH} 8.0), 300 \mathrm{mM} \mathrm{KCl}$ $1 \mathrm{mM}$ tris(2-carboxyethyl)phosphine (TCEP), and lysed using a C-5 Emulsiflex high-pressure homogenizer (Avestin). Lysates were then clarified and passed through two Histrap FF $5 \mathrm{ml}$ columns in series (GE Healthcare). Protein was eluted using a gradient from 15 to $500 \mu \mathrm{M}$ imidazole. Fast protein liquid chromatography was performed on either an NGC Scout system (Bio-Rad) or an ÄKTA Start (GE Healthcare). Eight milligrams of recombinant $6 \mathrm{xHis-tagged} \mathrm{TEV} \mathrm{was} \mathrm{added} \mathrm{to}$ eluted protein followed by dialysis into $50 \mathrm{mM}$ Tris (pH 8.0), $200 \mathrm{mM} \mathrm{NaCl}, 1 \mathrm{mM}$ TCEP, and $4 \mathrm{mM}$ EDTA. The protein was again passed through HisTrap FF columns in order to separate cleaved protein from TEV as well as the cleaved 6xHis tag. The cleavage step was only performed on protein which would be used for NMR or FP, but the protein used for TR-FRET did not have the $6 \mathrm{xHis}$ tag removed. The protein was then further purified by gel filtration using a HiLoad 16/600 Superdex 200 PG (GE Healthcare). Size exclusion was performed in $25 \mathrm{mM}$ MOPS (pH 8.0), $300 \mathrm{mM} \mathrm{KCl,} 1 \mathrm{mM}$ TCEP, and $1 \mathrm{mM}$ EDTA buffer. Protein was then dialyzed into $25 \mathrm{mM} 3$-( $N$-morpholino)propanesulfonic acid (MOPS) (pH 7.4), $25 \mathrm{mM} \mathrm{KCl}$, and $1 \mathrm{mM}$ EDTA buffer. Protein purity in excess of $95 \%$ was determined by gradient $4-20 \%$ sodium dodecyl sulfate-polyacrylamide gel electrophoresis analysis (NuSep). Protein concentration was determined using $\varepsilon_{280}=$ $12,045 \mathrm{M}^{-1} \mathrm{~cm}^{-1}$.

${ }^{15} \mathrm{~N}$-labeled protein was grown in M9 minimal media containing $99 \%{ }^{15} \mathrm{NH}_{4} \mathrm{Cl}$ (Cambridge Isotope Laboratories) as the sole nitrogen source. For this growth, cells were grown at $37^{\circ} \mathrm{C}$ and $180 \mathrm{rpm}$ until an $\mathrm{OD}_{600}$ of approximately 1.0 was reached. At this point, the temperature was dropped to $22^{\circ} \mathrm{C}$ for $1 \mathrm{~h}$. Following cool down period, protein expression was induced by the addition of $500 \mu \mathrm{M}$ IPTG during which induction cells remained at $22^{\circ} \mathrm{C}$. Protein expression and purification was then accomplished utilizing the same protocol as outlined above.

Delipidation of PPAR $\boldsymbol{\gamma}$. To delipidate PPAR $\gamma$ LBD, purified protein was diluted to $0.8 \mathrm{mg} \mathrm{ml}^{-1}$ and batched with Lipidex 1000 (Perkin-Elmer) at an equal volume. This mixture was batched for $1 \mathrm{~h}$ at $37^{\circ} \mathrm{C}$ and $100 \mathrm{rpm}$. Immediately following this treatment, protein was pulled through a gravity column by syringe. To increase 
yield, it was found that the speed of elution was important; protein could not remain on the resin at room temperature in excess of $3 \mathrm{~min}$. Two more column volumes of pre-warmed $25 \mathrm{mM}$ MOPS, $25 \mathrm{mM} \mathrm{KCl}$, and $1 \mathrm{mM}$ EDTA were also pulled through in the same manner. Quality of delipidation was then estimated by ${ }^{19} \mathrm{~F}$ NMR, and loss of lipid can be most easily detected by a reduction in the peak at $-84.1 \mathrm{ppm}$. When non-delipidated protein is used in this report it is labeled as bound to $E$. coli lipids.

Site-directed mutagenesis. Mutations in PPAR $\gamma$ LBD were generated using the Quikchange Lightning site-directed mutagenesis kit (Agilent) using primers listed in Supplementary Table 6. The presence of expected mutations and absence of spurious mutations was confirmed by Sanger sequencing (Eurofins).

Preparation of NMR samples. NMR samples were prepared to a final concentration of $150 \mu \mathrm{M}$ protein in $470 \mu \mathrm{L}$ volume containing $10 \% \mathrm{D}_{2} \mathrm{O}$. Addition of ligand was done in two separate injections of compound to reduce precipitation. Injections were spaced 30-60 min apart to allow time for binding. All ligands were dissolved in $\mathrm{D}_{6}$-dimethylsulfoxide (DMSO), with the exception of GQ-16, which was dissolved in $\mathrm{D}_{7}$-dimethylformamide (DMF). Deuterated solvents were obtained from Cambridge Isotope Laboratories Inc. and were at least 99\% isotopically pure. Final concentrations of ligand for samples of PPAR $\gamma^{\mathrm{K} 502 \mathrm{C}}$-BTFA were $1.25 \mathrm{x}$ ligand to protein $(187.5 \mu \mathrm{M})$, with the exception of troglitazone, pioglitazone, and ciglitazone, which were loaded to $2.0 \mathrm{x}(300 \mu \mathrm{M})$ due to poor binding affinity. In samples of PPAR $\gamma^{\mathrm{C} 313 \mathrm{~A}, \mathrm{~K} 502 \mathrm{C}}$-BTFA, ligand concentration was $1.1 \mathrm{x}$ to protein $(165 \mu \mathrm{M})$, with the exception of troglitazone, pioglitazone, and ciglitazone, which were loaded to $1.5 \mathrm{x}(225 \mu \mathrm{M})$. To decrease the likelihood of labeling the single native cysteine (C313) in PPAR $\gamma^{\mathrm{K} 502 \mathrm{C}}-\mathrm{BTFA}$, we first loaded ligands into the protein and then labeled with BTFA since bound ligands would restrict access to $\mathrm{C} 313$ for all experiments involving PPAR $\gamma^{\mathrm{K} 502 \mathrm{C}}$-BTFA. In both cases, the ligand concentration in DMSO was controlled to maintain a constant volume of DMSO or DMF addition to the sample $\left(8.80 \mu \mathrm{L}\right.$ for PPAR $\gamma^{\mathrm{K} 502 \mathrm{C}_{-} \mathrm{BTFA}}$ and $7.76 \mu \mathrm{L}$ for

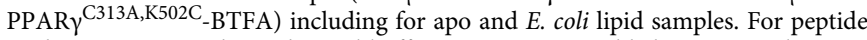
studies, $1 \mathrm{mM}$ peptide in identical buffer to protein was added at a 2:1 molar ratio (final concentration $300 \mu \mathrm{M}$ peptide and $150 \mu \mathrm{M}$ protein). For non-covalent ligands, samples were labeled with 2.0x BTFA after the addition of ligand. For covalent ligands, samples were labeled with 10x BTFA following preincubation of T0070907 or GW9662; and addition of 1.5 and 2 ligand molar ratios yielded very similar spectra, indicating complete covalent modification of $\mathrm{C} 313$ and likely no bonding to K502C (Supplementary Fig. 5). After the addition of BTFA, protein was incubated for 30-60 min and then buffer exchanged at least $100 \mathrm{x}$ using $10 \mathrm{kDa}$ Amicon Ultra-15 concentrators (Merck Millipore) to remove excess unbound BTFA. Following this buffered $\mathrm{D}_{2} \mathrm{O}$ was added.

Variable $\mathrm{D}_{2} \mathrm{O}$ NMR samples were prepared by buffer exchanging protein samples $>100 \mathrm{x}$ into $25 \mathrm{mM}$ MOPS, $25 \mathrm{mM} \mathrm{KCl}, 1 \mathrm{mM}$ EDTA (pD 7.4) buffer prepared in either 50 or $100 \% \mathrm{D}_{2} \mathrm{O}$ using Amicon Ultra-4 $10 \mathrm{kDa}$ centrifugal filters (EMD Millipore). One hundred percent $\mathrm{D}_{2} \mathrm{O}$ buffer was adjusted to read $\mathrm{pD} 7.4$ $\left(\mathrm{pH} \mathrm{7.0}{ }^{45}\right)$, and then mixed with appropriate amounts of $\mathrm{H}_{2} \mathrm{O}$ buffer that had been adjusted to read $\mathrm{pH}$ 7.4. Following this, ligands were added to the appropriate concentration as usual. The samples which contained only $10 \% \mathrm{D}_{2} \mathrm{O}$ were prepared as usual with the standard addition of $10 \%$ buffered $\mathrm{D}_{2} \mathrm{O}$ to the final sample. Samples of PPAR $\gamma^{\mathrm{K} 502 \mathrm{C}}$-BTFA were already loaded with ligand and labeled appropriately with BTFA prior to exchange into deuterated buffers.

FP assay. FP peptide binding assays were performed by plating a mixture of $50 \mathrm{nM}$ peptide with an N-terminal FITC tag, 12-point serial dilutions of PPAR $\gamma$-LBD (wt, PPAR $\gamma^{K 502 C}$-BTFA, PPAR $\gamma$ K502C, or PPAR $\gamma$ C313A,K502C - BTFA), and PPAR $\gamma$ ligands from $50 \mu \mathrm{M}$ to $24 \mathrm{nM}$. PPAR $\gamma$-LBD and PPAR $\gamma$ ligands were added at a 1:1 ratio. This mixture was added to wells of low-volume 384-well black plates (Grenier Bio-one, catalog number 784076) to a final volume of $16 \mu \mathrm{L}$. Peptides were synthesized by Lifetein LLC (Somerset, NJ, USA) for the for MED1 peptide, sequence: NTKNHPMLMNLLKDNPAQD; and the NCoR peptide, sequence: GHSFADPASNLGLEDIIRKALMG (2251-2273). Other peptides were purchased from ThermoFisher (Waltham, MA, USA) for MED1 peptide, sequence: NTKNHPMLMNLLKDNPAQD (catalog number PV4549); CBP peptide, sequence: AASKHKQLSELLRGGSGSS (catalog number PV4596); and SMRT, sequence: HASTNMGLEAIIRKALMGKYDQW (catalog number PV4424). All dilutions were made in $25 \mathrm{mM}$ MOPS ( $\mathrm{pH} 7.4$ ), $25 \mathrm{mM} \mathrm{KCl}, 1 \mathrm{mM}$ EDTA, $0.01 \%$ fatty-acid-free bovine serum albumin (BSA) (EMD Millipore, catalog number 126575), $0.01 \%$ Tween, and $5 \mathrm{mM}$ TCEP. Assay titrations were performed in duplicate. Plates were incubated in the dark at room temperature for $2 \mathrm{~h}$ before being read on a Synergy $\mathrm{H} 1$ microplate reader (BioTek). FP was measured by excitation at $485 \mathrm{~nm} / 20 \mathrm{~nm}$ and emission at $528 \mathrm{~nm} / 20 \mathrm{~nm}$ for FITC. Data were fit using nonlinear regression (agonist vs. response - variable slope 4 parameters) in Prism 7.0b. For FP, TR-FRET, and Fluormone competitive binding assays, we did two technical replicates and repeated these experiments independently in the lab for once (Fluormone) or 2 or more times FP and TR-FRET. We chose these number of technical replicates and independent experiment replicates based on our experience with the limited variability inherent in these biochemical assays
Time-resolved Förster resonance energy transfer assay. TR-FRET peptide recruitment assays were performed by plating a mixture of $8 \mathrm{nM} 6 \mathrm{xHis-PPAR} \gamma$ LBD (wt, PPAR $\gamma^{\mathrm{K} 502 \mathrm{C}}$-BTFA, or PPAR $\gamma^{\mathrm{C} 313 \AA, K 502 \mathrm{C}}$-BTFA), $0.9 \mathrm{nM}$ LanthaScreen Elite Tb-anti-His antibody (LifeTechnologies catalog number PV5863), $200 \mathrm{nM}$ peptide ( $\mathrm{N}$ terminally biotinylated and $\mathrm{C}$ terminally amidated), $400 \mathrm{nM}$ streptavidin-d2 (Cisbio, catalog number 610SADLB), and 12-point serial dilutions of PPAR $\gamma$ ligands from $50 \mu \mathrm{M}$ to $1 \mathrm{pM}$. This mixture was added to wells of lowvolume 384-well black plates (Grenier Bio-one, catalog number 784076) to a final volume of $20 \mu \mathrm{L}$. Peptides were synthesized by Lifetein LLC (Somerset, NJ, USA) for MED1 peptide; sequence: VSSMAGNTKNHPMLMNLLKDNPAQ; and NCoR peptide, sequence: GHSFADPASNLGLEDIIRKALMG (2251-2273). All dilutions were made in $25 \mathrm{mM}$ MOPS (pH 7.4), $25 \mathrm{mM} \mathrm{KCl}, 1 \mathrm{mM}$ EDTA, $0.01 \%$ fatty-acid free BSA (EMD Millipore, catalog number 126575), 0.01\% Tween, and $5 \mathrm{mM}$ TCEP. Assay titrations were performed in duplicate. Plates were incubated in the dark at room temperature for $2 \mathrm{~h}$ before being read on a Synergy $\mathrm{H} 1$ microplate reader (BioTek). TR-FRET was measured by excitation at $330 \mathrm{~nm} / 80 \mathrm{~nm}$ and emission at $620 \mathrm{~nm} / 10 \mathrm{~nm}$ for terbium and $665 \mathrm{~nm} / 8 \mathrm{~nm}$ for d2. Change in TR-FRET was calculated by $665 \mathrm{~nm} / 620 \mathrm{~nm}$ ratio.

Data were fit using nonlinear regression (agonist vs. response - variable slope 4 parameters) in Prism 7.0b. Outliers were automatically detected using Prism 7.0b's implementation of the ROUT method ${ }^{46}$ and excluded from the curve fitting (20 out of 2260 total data points were flagged as outliers). However, all data points, including detected outliers, were included in figure graphs. In cases where curve fitting failed, the TR-FRET value nearest the calculated free ligand concentration in the NMR experiment was used; otherwise, the TR-FRET value at the calculated free NMR ligand concentration was calculated using the fitted curve. These TR-FRET ratio values were used to correlate with NMR chemical shift values. One ligand, nTZDpa, showed a biphasic TR-FRET curve. This could be due to several factors including ligand aggregation ${ }^{47}$, absorption interference in the TR-FRET assay (nTZDpa contains an indole group that could in principle cause interference), or alternate site binding effects ${ }^{18}$; we therefore utilized the value of the TR-FRET ratio for nTZDpa based on a fit that did not include the last two concentrations (10 and $50 \mu \mathrm{M})$, so as to exclude most effects of the second transition. In addition, the $50 \mu \mathrm{M}$ SR2088 point was not run because we did not have sufficient ligand. All error bars are standard deviation.

Fluormone competitive binding assays. PPAR $\gamma$ ligand inhibition constants $\left(K_{\mathrm{i}}\right)$ were measured using a protocol adapted from LanthaScreen TR-FRET PPAR $\gamma$ competitive binding assay (Invitrogen, catalog number PV4894). Assay was performed by plating a mixture of $8 \mathrm{nM} 6 \mathrm{xHis}-\mathrm{PPAR} \gamma-\mathrm{LBD}, 2.5 \mathrm{nM}$ LanthaScreen Elite Tb-anti-His antibody, $5 \mathrm{nM}$ LanthaScreen Fluormone Pan-PPAR Green (Invitrogen, catalog number PV4896), and 12-point serial dilutions of PPAR $\gamma$ ligands from $50 \mu \mathrm{M}$ to $140 \mathrm{fM}$. This mixture was added to wells of low-volume 384-well black plates (Grenier Bio-one) to a final volume of $16 \mu \mathrm{L}$. All dilutions were made in $25 \mathrm{mM}$ MOPS (pH 7.4), $25 \mathrm{mM} \mathrm{KCl,} 1 \mathrm{mM}$ EDTA, $0.01 \%$ fatty-acid free BSA (EMD Millipore), $0.01 \%$ Tween, and $5 \mathrm{mM}$ TCEP. Assay titrations were performed in duplicate. Plates were incubated in the dark for $2 \mathrm{~h}$ at room temperature before being read on a Synergy H1 microplate reader (BioTek). TR-FRET was measured by excitation at $330 \mathrm{~nm} / 80 \mathrm{~nm}$ and emission at $495 \mathrm{~nm} / 10 \mathrm{~nm}$ for terbium and $520 \mathrm{~nm} / 25 \mathrm{~nm}$ for Fluormone. Change in TR-FRET was calculated by $520 \mathrm{~nm} / 495 \mathrm{~nm}$ ratio. Nonlinear curve fitting was performed using Prism 7.0b (Graphpad Software Inc.) as described above for the TR-FRET data, including manual exclusion of highest two concentrations for nTZDpa. Thirty of the 1224 total data points for all three proteins (wt, PPAR $\gamma^{\mathrm{K} 502 \mathrm{C}}-\mathrm{BTFA}$, and PPAR $\gamma$ C313A, K502C-BTFA) were automatically excluded by Prism in the fits.

$\boldsymbol{K}_{\mathbf{i}}$ calculation. The inhibition constant for each PPAR $\gamma$ ligand was calculated by applying a corrected Cheng-Prusoff ${ }^{48,49}$

$$
K_{\mathrm{i}}=\frac{(\mathrm{Lb})\left(\mathrm{IC}_{50}\right)\left(K_{\mathrm{d}}\right)}{(\mathrm{Lo})(\mathrm{Ro})+\mathrm{Lb}\left(\mathrm{Ro}-\mathrm{Lo}+\mathrm{Lb}-K_{\mathrm{d}}\right)},
$$

where $\mathrm{IC}_{50}$ is the concentration of the ligand that produces $50 \%$ displacement of the Fluormone tracer, Lo is the concentration of Fluormone in the assay $(5 \mathrm{nM})$, and $K_{\mathrm{d}}$ is the binding constant of Fluormone to wt or the two BTFA-labeled mutants, Ro is the total receptor concentration, and Lb is the concentration of bound Fluormone in the assay with no addition of test ligand. The affinity of Fluormone for the two BTFA-labeled mutant proteins was determined via TRFRET by titration of Fluormone into each mutant bound to Elite Tb-anti-His antibody. Dissociation constants of Fluormone for wt was measured as $7.9 \pm 0.2$ for PPAR $\gamma$ LBD, and the variants were measured as $26 \pm 3 \mathrm{nM}$ for PPAR $\gamma^{\mathrm{K} 502 \mathrm{C}}-\mathrm{BTFA}$ and $44 \pm 4 \mathrm{nM}$ for PPAR $\gamma^{\mathrm{C} 313 \mathrm{~A}, \mathrm{~K} 502 \mathrm{C}}$-BTFA and $12 \pm 1 \mathrm{nM}$ PPAR $\gamma \mathrm{C} 313 \mathrm{~A}$.

NMR spectroscopy. Acquisition of spectra was performed using a Bruker 700 $\mathrm{MHz}$ NMR system equipped with a QCI-F cryoprobe. Chemical shifts were calibrated using an internal separated $\mathrm{KF}$ reference in $20 \mathrm{mM} \mathrm{KPO}_{4}(\mathrm{pH} 7.4)$ and 50 $\mathrm{mM} \mathrm{KCl}$ contained in a coaxial tube inserted into the NMR sample tube. KF was set to be $-119.522 \mathrm{ppm}$, which is the shift of the KF signal with respect to the ${ }^{19} \mathrm{~F}$ basic transmitter frequency for the instrument $(658.8462650 \mathrm{MHz})$ at $298.2 \mathrm{~K}$, the temperature at which samples were run. Routine $1 \mathrm{D}$ fluorine spectra were acquired 
utilizing the zgfhigqn.2 pulse program (Bruker Topspin 3.5), which consists of a $90^{\circ}$ pulse followed by acquisition with proton decoupling (acquisition $=0.7 \mathrm{~s}$ ). Settings were $\mathrm{D} 1=1.2 \mathrm{~s}, \mathrm{AQ}=0.82 \mathrm{~s}$. Approximately 500 to 4000 transients were collected. Saturation transfer experiments were carried out using the stddiff pulse program. Settings were $\mathrm{D} 1=1.6 \mathrm{~s}, \mathrm{AQ}=0.6 \mathrm{~s}$. For some experiments, the total duration of the saturating pulse (Gaus1.1000, $54.52 \mathrm{~dB}, 50 \mathrm{~ms}$ ) was $1.6 \mathrm{~s}\left(\mathrm{D}_{2} \mathrm{O}\right)$ and the location of the saturating pulse was varied. In other experiments, the duration of the saturating pulse was varied, with the saturating pulse location held constant and the rate of exchange was fit using equation 50 found in a previous publication $^{50}$. An off resonance selective saturating pulse was used to determine the peak intensity at time $t=0$ and $R_{1}$ was determined experimentally (Supplementary Table 5). Fits of the saturation transfer data were accomplished with a single free parameter using these experimentally determined values for initial intensity and $R_{1}$ $\left(2.7 \mathrm{~s}^{-1}\right.$ used for all fits except GW9662 which used $\left.2.4 \mathrm{~s}^{-1}\right)$. Transverse and longitudinal relaxation lifetimes $\left(T_{1}\right.$ and $\left.T_{2}\right)$ were determined by fitting data acquired using an inversion recovery experiment (Bruker pulse program tlir) and cpmg pulse sequence (Bruker cpmg) in Prism 7.0b (Graphpad Software Inc.) using standard formulas. Spectra were deconvoluted in an objective manner with models chosen statistically by a fitting program ${ }^{32}$. All fits were carried out in the same manner with the same settings in the fitting program, except where noted. Relative phase of fitted peaks was allowed to vary slightly $(\pi / 50 \mathrm{rad})$ to accommodate imperfect phasing of these broad signals. The fitting algorithm ${ }^{32}$ assumes Lorentzian lineshapes of similar phase. Intermediate exchange effects and field inhomogeneity are likely present in some of these spectra, which will result in inaccuracies in the fitted models; however, notwithstanding these limitations, the deconvolution method provides an objective view of the possible underlying spectral structure and populations. Two-dimensional $\left[{ }^{1} \mathrm{H},{ }^{15} \mathrm{~N}\right]$ TROSY-HSQC NMR data were obtained using the trosyf3gpphsi19.2 pulse program. Select NMR spectra were replicated in two different ways: (1) Some NMR samples were measured via NMR initially and then days to weeks later to determine if certain parts of the spectrum changed. Any changes would indicate that non-reversible processes contribute to that part of the signal, such as unfolding or degradation of the protein. (2) Some spectra were run twice utilizing protein from the same batch as utilized for the first spectra or from an entirely different protein preparation.

Molecular dynamics simulations. Residues in our physical protein constructs used in NMR that were missing in crystal structures (except the N-terminal glycine, which is an unnatural vestige of the cleaved His tag) were added using the modeler ${ }^{51,52}$ within Chimera ${ }^{53}$ and a PDB file was saved. This PDB file was then submitted to the $\mathrm{h}++$ server $^{54}$ (http://biophysics.cs.vt.edu/H++) to determine the

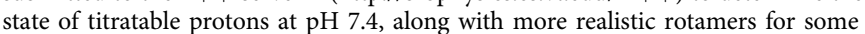
residues. This $\mathrm{h}++$ PDB file was then given AMBER names for the various protonation states of histidine determined by $\mathrm{h}++$ using pdb4amber (AmberTools $14^{55}$ ). PDB files of cysteine-BTFA residue was created through modification of a cysteine residue in chimera. This PDB file was then submitted to the RED server ${ }^{56}$ for RESP ${ }^{57}$ charge derivation and geometry optimization. RESP values for the cysteine backbone of these modified residues were constrained to match AMBER cysteine residue values as part of the input to the RED server. In a manner similar to that outlined in sections $2-3$ in tutorial 5 on the ambermd.org site (http://ambermd.org/tutorials/basic/tutorial5/), the output mol2 file was then used to prepare an ac file and then a prepin file containing the same RESP-derived charges (Supplementary Note 2) and two force modification files. The AMBER parameter database derived frcmod file (Supplementary Note 3) was loaded after the $\mathrm{GAFF}^{58}$ parameter database derived frcmod file (Supplementary Note 4) within Tleap in order to use AMBER parameters where possible for the cysteine-BTFA residue. Tleap was then used to generate parameter and coordinate files using both ff $14 \mathrm{SB}^{59}$ and GAFF values. A truncated octahedron solvation cell with boundaries at least $10 \AA$ from any protein atom was built with TIP3 $\mathrm{P}^{60}$ water. The system was neutralized with $\mathrm{Na}^{+}$ions and $\mathrm{K}^{+}$and $\mathrm{Cl}^{-}$atoms were added to $50 \mathrm{mM}$. Joung and Cheatham ${ }^{61}$ ion parameters were used. Minimization (imin $=1$ ) and equilibration was carried out in nine steps with non-bonded cutoff (cut) set to $8 \AA \AA$ and with the equilibrations carried out at $310 \mathrm{~K}$. First, steepest descent minimization $($ ntmin $=2)$ with strong restraints (restraint_wt $=5 \mathrm{kcal} \mathrm{mol}^{-1} \AA^{-2}$ ) on protein heavy atoms for 2000 steps was used followed by NTV MD with shake, the same restraints and $1 \mathrm{fs}$ steps for $15 \mathrm{ps}$. Next, two rounds of 2000 steps of steepest descent minimization with progressively relaxed restraints (restraint_wton protein heavy atoms for 2000 steps was used followed by 2 and $0.1 \mathrm{kcal} \mathrm{mol}^{-1} \AA^{-2}$ ) followed by a round without restraints. This was followed by three rounds of NTP MD with shake $(5,10$, and $10 \mathrm{ps}$ in duration), and protein heavy atom restraints of $1,0.5$, and $0.5 \mathrm{kcal} \mathrm{mol}^{-1} \AA^{-2}$. A final unrestrained NTP MD simulation was then run for $200 \mathrm{ps}$ with $2 \mathrm{fs}$ steps. The final restart file from this process was used along with a hydrogen mass repartitioned parameter file (modified using parmed) to run new simulations with new randomized atomic velocities using 4 fs steps at $310 \mathrm{~K}$. Analysis was carried out using CPPTRA ${ }^{62}$. All production simulations were carried out using pmemd.cuda or pmemd.cuda.MPI.

The S enantiomer of GW1929, which was used in NMR and TR-FRET, was built using Maestro (Schrödinger LLC). The pyridine ring nitrogen of GW1929 was the only atom with a predicted $\mathrm{pKa}$ near 7.4 (calculated $7.56 \pm 1.12$ ) using the EPIK module (Schrödinger LLC). We chose to model this nitrogen as deprotonated. There is no crystal structure for GW1929 bound to PPAR $\gamma$; however, there is crystal structure for GI262570, which is bound to PPAR $\gamma$ LBD in the 1FM9 crystal structure. GI262570 is identical to GW1929 for about 2/3 of the molecule. GW1929 was docked into the 1FM9 crystal structure with GI262570 removed using AutoDock Vina ${ }^{63}$. The best scoring docked binding mode overlaid well with GI262570. In this docked model in the helix 12-interacting region, the two ligands themselves are identical. RESP charges for GW1929 were derived using the RED server and force modification files were generated using GAFF parameters. 1FM9 was modified (or not) to incorporate cysteine-BTFA (parameterized as described above) in place of K502 and docked with GW1929 in a similar way to that described above to build PPAR $\gamma$ K502C-BTFA and PPAR $\gamma$ bound to GW1929.

1PRG chain B was used to create the build for apo and 3BOR chain B was used to build T0070907-bound PPAR $\gamma$ LBD. 3BOR contains GW9662 which differs from T0070907 by one atom. T0070907 has a nitrogen in place of a carbon atom in one of the ligand rings. This change was made in chimera and the ligand parameterized and incorporated into the structure as described above for BTFA. NCoR (same sequence as used in NMR and TR-FRET including N-terminal acetylation and C-terminal amidation) was added to these builds utilizing chimera using the following procedure. The core helix structure from NCoR (from 2OVM) was aligned to SMRT on a PPAR $\alpha$ SMRT structure (1KKQ), and then apo PPAR $\gamma$ chain B (1PRG) was aligned to PPARa and the PPARa/SMRT structure deleted leaving the aligned NCoR on apo PPAR $\gamma$ chain $\mathrm{B}$. A similar procedure was used with the 3BOR structure to create T0070907 co-bound with NCoR on PPAR $\gamma$. These PDBs were then used to create the final solvated, minimized, and equilibrated structure in a manner similar to that described above.

All simulations were run with settings shown in Supplementary Note 1 including utilization of SHAKE ${ }^{64}$, with variability in the frequency of writing various files to disk. $K$-means clustering was performed and representative structures from these structures were outputted by CPPTRAJ ${ }^{62}$.

Data availability. Data files for TR-FRET, Fluormone competitive binding assays, and FP are publically available at https://osf.io/rqdpz/. Any other datasets generated during and/or analyzed during the current study are available from the corresponding author on reasonable request.

Received: 22 October 2017 Accepted: 6 April 2018

Published online: 04 May 2018

\section{References}

1. Steinmetz, A. C., Renaud, J. P. \& Moras, D. Binding of ligands and activation of transcription by nuclear receptors. Annu. Rev. Biophys. Biomol. Struct. 30, 329-359 (2001).

2. Overington, J. P., Al-Lazikani, B. \& Hopkins, A. L. How many drug targets are there? Nat. Rev. Drug. Discov. 5, 993-996 (2006).

3. Nettles, K. W. \& Greene, G. L. Ligand control of coregulator recruitment to nuclear receptors. Annu. Rev. Physiol. 67, 309-333 (2005).

4. Brzozowski, A. M. et al. Molecular basis of agonism and antagonism in the oestrogen receptor. Nature 389, 753-758 (1997).

5. Shiau, A. K. et al. The structural basis of estrogen receptor/co-activator recognition and the antagonism of this interaction by tamoxifen. Cell $\mathbf{9 5}$, 927-937 (1998).

6. Bourguet, W., Ruff, M., Chambon, P., Gronemeyer, H. \& Moras, D. Crystal structure of the ligand-binding domain of the human nuclear receptor RXRalpha. Nature 375, 377-382 (1995).

7. $\mathrm{Xu}, \mathrm{H}$. E. et al. Structural basis for antagonist-mediated recruitment of nuclear co-repressors by PPARa. Nature 415, 813-817 (2002).

8. Bain, D. L., Heneghan, A. F., Connaghan-Jones, K. D. \& Miura, M. T. Nuclear receptor structure: implications for function. Annu. Rev. Physiol. 69, 201-220 (2007).

9. Kroker, A. J. \& Bruning, J. B. Review of the structural and dynamic mechanisms of PPAR $\gamma$ partial agonism. PPAR Res. 2015, 816856 (2015).

10. Nagy, L. \& Schwabe, J. W. R. Mechanism of the nuclear receptor molecular switch. Trends Biochem. Sci. 29, 317-324 (2004).

11. Kojetin, D. J. \& Burris, T. P. Small molecule modulation of nuclear receptor conformational dynamics: implications for function and drug discovery. Mol. Pharmacol. 83, 1-8 (2013).

12. Choi, J. H. et al. Anti-diabetic drugs inhibit obesity-linked phosphorylation of PPARgamma by Cdk5. Nature 466, 451-456 (2010).

13. Marciano, D. P. D. P. et al. Pharmacological repression of PPAR $\gamma$ promotes osteogenesis. Nat. Commun. 6, 7443 (2015).

14. Wright, E. et al. Helix 11 dynamics is critical for constitutive androstane receptor activity. Structure 19, 37-44 (2011).

15. Zhang, J. et al. DNA binding alters coactivator interaction surfaces of the intact VDR-RXR complex. Nat. Struct. Mol. Biol. 18, 556-563 (2011).

16. Johnson, B. A. et al. Ligand-induced stabilization of $\operatorname{PPAR} \gamma$ monitored by NMR spectroscopy: implications for nuclear receptor activation. J. Mol. Biol. 298, 187-194 (2000). 
17. Hughes, T. S. et al. Ligand and receptor dynamics contribute to the mechanism of graded PPAR $\gamma$ agonism. Structure 20, 139-150 (2012).

18. Hughes, T. S. et al. An alternate binding site for PPAR $\gamma$ ligands. Nat. Commun. 5, 3571 (2014)

19. Berger, J. P. et al. Distinct properties and advantages of a novel peroxisome proliferator-activated protein [gamma] selective modulator. Mol. Endocrinol. 17, 662-676 (2003).

20. Nolte, R. T. et al. Ligand binding and co-activator assembly of the peroxisome proliferator-activated receptor-gamma. Nature 395, 137-143 (1998)

21. Sharaf, N. G. \& Gronenborn, A. M. (19)F-modified proteins and (19)Fcontaining ligands as tools in solution NMR studies of protein interactions. Methods Enzymol. 565, 67-95 (2015).

22. Kim, T. H. et al. The role of ligands on the equilibria between functional states of a G protein-coupled receptor. J. Am. Chem. Soc. 135, 9465-9474 (2013).

23. Nygaard, R. et al. The dynamic process of $\beta 2$-adrenergic receptor activation. Cell 152, 532-542 (2013).

24. Manglik, A. et al. Structural insights into the dynamic process of $\beta 2$ adrenergic receptor signaling. Cell 161, 1101-1111 (2015).

25. Liu, J. J., Horst, R., Katritch, V., Stevens, R. C. \& Wüthrich, K. Biased signaling pathways in $\beta 2$-adrenergic receptor characterized by 19F-NMR. Science 335, 1106-1110 (2012).

26. Kitevski-LeBlanc, J. L. \& Prosser, R. S. Current applications of 19F NMR to studies of protein structure and dynamics. Prog. Nucl. Magn. Reson. Spectrosc. 62, 1-33 (2012).

27. Lehmann, J. M. et al. An antidiabetic thiazolidinedione is a high affinity ligand for peroxisome proliferator-activated receptor gamma (PPAR gamma). J. Biol. Chem. 270, 12953-12956 (1995).

28. Brown, K. K. et al. A novel $N$-aryl tyrosine activator of peroxisome proliferator-activated receptor- $\gamma$ reverses the diabetic phenotype of the Zucker diabetic fatty rat. Diabetes 48, 1415-1424 (1999).

29. Lee, G. et al. T0070907, a selective ligand for peroxisome proliferator-activated receptor- $\gamma$ functions as an antagonist of biochemical and cellular activities. $J$. Biol. Chem. 277, 19649-19657 (2002).

30. Brust, R. et al. Modification of the orthosteric PPAR $\gamma$ covalent antagonist scaffold yields an improved dual-site allosteric inhibitor. ACS Chem. Biol. 12, 969-978 (2017).

31. Liberato, M. V. et al. Medium chain fatty acids are selective peroxisome proliferator activated receptor (PPAR) gamma activators and Pan-PPAR partial agonists. PLoS ONE 7, e36297 (2012).

32. Hughes, T. S., Wilson, H. D., De Vera, I. M. S. \& Kojetin, D. J. Deconvolution of complex 1D NMR spectra using objective model selection. PLOS ONE 10, e0134474 (2015).

33. Evanics, F. et al. Tryptophan solvent exposure in folded and unfolded states of an SH3 domain by 19F and 1H NMR. Biochemistry 45, 14120-14128 (2006).

34. Bruning, J. B. et al. Partial agonists activate PPAR $\gamma$ using a Helix 12 independent mechanism. Structure 15, 1258-1271 (2007).

35. Evanics, F., Kitevski, J. L., Bezsonova, I., Forman-Kay, J. \& Prosser, R. S. ${ }^{19}$ F NMR studies of solvent exposure and peptide binding to an $\mathrm{SH} 3$ domain. Biochim. Biophys. Acta 1770, 221-230 (2007).

36. Henzler-Wildman, K. \& Kern, D. Dynamic personalities of proteins. Nature 450, 964-972 (2007).

37. Hwan, Bae et al. Mechanistic elucidation guided by covalent inhibitors for the development of anti-diabetic PPAR $\gamma$ ligands. Chem. Sci. 7, 5523-5529 (2016).

38. Nielsen, R. et al. Genome-wide profiling of PPAR $\gamma$ :RXR and RNA polymerase II occupancy reveals temporal activation of distinct metabolic pathways and changes in RXR dimer composition during adipogenesis. Genes Dev. 22, 2953-2967 (2008).

39. Song, Y. \& Dai, L. L. The shear viscosities of common water models by non-equilibrium molecular dynamics simulations. Mol. Simul. 36, 560-567 (2010).

40. Batista, M. R. B. \& Martínez, L. Conformational diversity of the helix 12 of the ligand binding domain of PPAR $\gamma$ and functional implications. J. Phys. Chem. B 119, 15418-15429 (2015).

41. Perissi, V. et al. Molecular determinants of nuclear receptor-corepressor interaction. Genes Dev. 13, 3198-3208 (1999).

42. Ye, L., Van Eps, N., Zimmer, M., Ernst, O. P. \& Scott Prosser, R. Activation of the A2A adenosine G-protein-coupled receptor by conformational selection. Nature 533, 1-15 (2016).

43. Staus, D. P. et al. Allosteric nanobodies reveal the dynamic range and diverse mechanisms of G-protein-coupled receptor activation. Nature 535, 448-452 (2016).

44. Kojetin, D. J. et al. Structural mechanism for signal transduction in RXR nuclear receptor heterodimers. Nat. Commun. 6, 8013 (2015).

45. Glasoe, P. K. \& Long, F. A. Use of glass electrodes to measure acidities in deuterium oxide. J. Phys. Chem. B. 64, 1-3 (1952).

46. Motulsky, H. J. \& Brown, R. E. Detecting outliers when fitting data with nonlinear regression-a new method based on robust nonlinear regression and the false discovery rate. BMC Bioinform. 7, 123 (2006).
47. Owen, S. C. et al. Colloidal drug formulations can explain "bell-shaped" concentration-response curves. ACS Chem. Biol. 9, 777-784 (2014).

48. Wei, S. et al. Active Pin 1 is a key target of all-trans retinoic acid in acute promyelocytic leukemia and breast cancer. Nat. Med. 21, 457-466 (2015).

49. Kenakin, T. P. Pharmacologic Analysis of Drug/Receptor Interaction 2nd edn (Raven, New York, 1993).

50. Helgstrand, M., Härd, T. \& Allard, P. Simulation of NMR pulse sequences during equilibrium and non-equilibrium chemical exchange. J. Biomol. NMR 18, 49-63 (2000)

51. Fernandez-Fuentes, N. \& Fiser, A. in Introduction to Protein Structure Predictiction: Methods and Algorithms (eds Rangwala, H. \& Karypis, G.) 279-298 (Wiley, 2010).

52. Šali, A. \& Blundell, T. L. Comparative protein modelling by satisfaction of spatial restraints. J. Mol. Biol. 234, 779-815 (1993).

53. Pettersen, E. F. et al. UCSF Chimera-a visualization system for exploratory research and analysis. J. Comput. Chem. 25, 1605-1612 (2004).

54. Anandakrishnan, R., Aguilar, B. \& Onufriev, A. V. H++ 3.0: automating pK prediction and the preparation of biomolecular structures for atomistic molecular modeling and simulations. Nucleic Acids Res. 40, 537-541 (2012).

55. Case, D. A. et al. Amber 14 (University of California, San Francisco, 2014).

56. Vanquelef, E. et al. R.E.D. Server: A web service for deriving RESP and ESP charges and building force field libraries for new molecules and molecular fragments. Nucleic Acids Res. 39, W511-W517 (2011).

57. Cornell, W. D., Cieplak, P., Bayly, C. I. \& Kollman, P. A. Application of RESP charges To calculate conformational energies, hydrogen bond energies, and free energies of solvation. J. Am. Chem. Soc. 115, 9620-9631 (1993).

58. Wang, J. M., Wolf, R. M., Caldwell, J. W., Kollman, P. A. \& Case, D. A. Development and testing of a general amber force field. J. Comput. Chem. 25, 1157-1174 (2004).

59. Maier, J. A. et al. ff14SB: improving the accuracy of protein side chain and backbone parameters from ff99SB. J. Chem. Theory Comput. 11, 3696-3713 (2015).

60. Jorgensen, W. L., Chandrasekhar, J., Madura, J. D., Impey, R. W. \& Klein, M. L. Comparison of simple potential functions for simulating liquid water. $J$. Chem. Phys. 79, 926-935 (1983).

61. Joung, I. S. \& Cheatham, T. E. Determination of alkali and halide monovalent ion parameters for use in explicitly solvated biomolecular simulations. J. Phys. Chem. B. 112, 9020-9041 (2008).

62. Roe, D. R. \& Cheatham, T. E. PTRAJ and CPPTRAJ: software for processing and analysis of molecular dynamics trajectory data. J. Chem. Theory Comput. 9, 3084-3095 (2013).

63. Trott, O. \& Olson, A. AutoDock Vina: improving the speed and accuracy of docking with a new scoring function, efficient optimization and multithreading. J. Comput. Chem. 31, 455-461 (2010).

64. Ryckaert, J. P., Ciccotti, G. \& Berendsen, H. J. C. Numerical integration of the Cartesian equations of motion of a system with constraints: molecular dynamics of $n$-alkanes. J. Comput. Phys. 23, 327-341 (1977).

\section{Acknowledgements}

We thank James Aramini at the City University of New York Advanced Science Research Center (CUNY ASRC) for assistance in setting up the saturation transfer difference, cpmg, and tlir experiments and Daniel R. Roe (NIH Laboratory of Computational Biology, NHLBI) for providing the minimization and equilibration script and simulation advice. NMR data presented herein were collected at the CUNY ASRC Biomolecular NMR Facility. This work was supported in part by National Institutes of Health (NIH) grants K99DK103116 (to T.S.H.), R00DK103116 (to T.S.H.), P20GM103546 (to T.H.S.), DK101871 (to D.J.K.), and DK105825 (to P.R.G.); and National Science Foundation (NSF) award 1359369 (PI Karbstein) that funds the SURF program at Scripps Florida.

\section{Author contributions}

T.S.H. and D.J.K. conceived the study and designed experiments. I.M.C. and M.D.N. prepared samples and performed experiments. T.S.H., I.M.S.dV., J.S., and J.F. prepared samples and performed preliminary experiments. Z.H. and T.S.H. built, performed, and analyzed the molecular simulations. Y.L. assisted with preliminary experiments. A.-L.B., Y.S., and T.M.K. synthesized ligands. H.R.-C. cloned and verified K502C plasmid. P.R.G. contributed to conception, provided key reagents, and characterization of ligand pharmacology. R.G.-M. and T.E.C. III assisted T.S.H. with molecular dynamics simulations and ligand parameterization. T.S.H. and D.J.K. analyzed data and wrote the manuscript with input from all authors.

\section{Additional information}

Supplementary Information accompanies this paper at https://doi.org/10.1038/s41467 018-04176-x.

Competing interests: The authors declare no competing interests. 
Reprints and permission information is available online at http://npg.nature.com/ reprintsandpermissions/

Publisher's note: Springer Nature remains neutral with regard to jurisdictional claims in published maps and institutional affiliations.

(c) (i) Open Access This article is licensed under a Creative Commons Attribution 4.0 International License, which permits use, sharing, adaptation, distribution and reproduction in any medium or format, as long as you give appropriate credit to the original author(s) and the source, provide a link to the Creative
Commons license, and indicate if changes were made. The images or other third party material in this article are included in the article's Creative Commons license, unless indicated otherwise in a credit line to the material. If material is not included in the article's Creative Commons license and your intended use is not permitted by statutory regulation or exceeds the permitted use, you will need to obtain permission directly from the copyright holder. To view a copy of this license, visit http://creativecommons.org/ licenses/by/4.0/.

(C) The Author(s) 2018 